\title{
Conflicto distributivo y política de ingresos en torno al tipo de cambio real en una economía semiindustrializada agroexportadora
}

\author{
FIRMENICH, MARIO EDUARDO \\ Estudios de Economía y Empresa \\ Universitat Oberta de Catalunya \\ Correo electrónico: firmenich@economistes.com
}

\begin{abstract}
RESUMEN
Se plantea un conflicto de tipo ricardiano. Terratenientes latifundistas producen bienes salario exportables y consumen bienes de lujo importados; industriales pequeños producen manufacturas no transables con costes hundidos importando bienes de capital; los asalariados consumen bienes exportables y manufacturas no transables.

Las cantidades se miden en unidades físicas y los precios en dinero fiduciario. La divisa internacional es fiduciaria. No existe equilibrio de economía pura. Equilibrio de precios no implica pleno empleo.

Según la gestión política, un incremento (reducción) del tipo de cambio real beneficia (perjudica) a exportadores, reduce (aumenta) el salario real y tiene efectos oscilantes en beneficios industriales, generando alianzas oscilantes de industriales con terratenientes o asalariados buscando un tipo de cambio real favorable en un juego de suma cero. La estabilización con el enfoque monetario de la balanza de pagos es imposible.

El modelo teórico formaliza la crisis estructural de economías periféricas que financian industrias tecnológicamente dependientes con exportaciones agrícolas.
\end{abstract}

Palabras clave: conflicto distributivo, tipo de cambio real, crecimiento desequilibrado. Clasificación JEL: O15; O24; O41.

MSC2010: 03C30; 37N40. 


\title{
Distributive conflict and income's policy around the real exchange rate in a semi industrialized agro- exporter economy
}

\begin{abstract}
A conflict of Ricardian type is arised. Landowners produce exportable salarygoods and consume imported luxury goods. Small industrialists produce nontradable manufactures with sunk costs by importing capital goods. Employees consume exportable goods and non-tradable manufactures.

Quantities are measured in physical units and prices in fiat money. The international currency is fiduciary. There is no equilibrium of pure economy. Price equilibrium does not imply full employment.

According to the political management, an increase (reduction) in the real exchange rate benefits (harms) exporters, reduces (increases) the real salary and has oscillating effects on industrial benefits, generating oscillating alliances of the industrialists, with eather landowners or workers, in order to achive a favorable real change rate in a zero sum game. Stabilization with the monetary approach to the balance of payments is impossible.

The theoretical model formalizes the structural crisis of peripheral economies that finance technologically dependent industries with agricultural exports.
\end{abstract}

Keywords: distributive conflict, real exchange rate, unbalanced growth.

JEL classification: O15; O24; O41.

MSC2010: 03C30; 37N40.

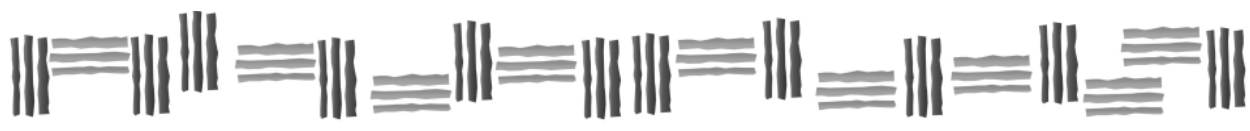




\section{Introducción.}

Este trabajo formaliza la dinámica desequilibrada de una economía semiindustrializada agroexportadora. Se plantea un conflicto de tipo ricardiano con tres clases sociales: terratenientes latifundistas, capitalistas industriales pequeños y medianos y asalariados. El modelo teórico puede ilustrar la crisis estructural de muchas economías periféricas, especialmente latinoamericanas, que dependen de sus exportaciones agrícolas para financiar un desarrollo industrial tecnológicamente dependiente.

El caso típico de la economía argentina ha sido tratado en múltiples trabajos, como Díaz Alejandro (1965), Porto (1975), Gueberoff (1977), Braun y Leonard (1981). La evidencia empírica histórica del caso argentino para las cuatro décadas que van desde los años '20 hasta los años '60 del S. XX ha sido bien recopilada y analizada por Díaz Alejandro (1975), aunque el autor no formaliza el modelo que represente esa dinámica.

En este trabajo se desarrolla un modelo teórico general sin formalizar ningún caso particular. A diferencia de otros trabajos, incluyendo todos los mencionados, la formalización que plantea este artículo se aleja de la economía matemática habitual y de los supuestos de la teoría neoclásica en particular.

La estructura del trabajo está organizada del siguiente modo. En primer lugar, se formaliza los sectores productivos, las preferencias de los consumidores y las condiciones de equilibrio en los mercados de dinero, agropecuario, industrial, laboral, del sector externo y las condiciones del equilibrio macroeconómico. En segundo lugar, se analizan los efectos distributivos de las variaciones del tipo de cambio real. En tercer lugar, se analizan los efectos de las variaciones del tipo de cambio real sobre la renta nacional en el corto plazo. En cuarto lugar, se analiza los efectos de las variaciones del tipo de cambio real en la dinámica del crecimiento económico. Por último, se exponen las conclusiones.

\section{Estructura básica del modelo y comportamiento de los agentes.}

\subsection{Los sectores productivos y sus respectivas tecnologías.}

Existe un sector productor de bienes primarios alimenticios y un sector productor de bienes manufacturados de consumo final. En ambos casos la tecnología se representa por funciones de producción técnicas medidas en unidades físicas ${ }^{1}$. El sector agropecuario está constituido por latifundios y el sector industrial por empresas pequeñas y medianas.

\footnotetext{
${ }^{1}$ La homogeneidad de las unidades de medida físicas de los factores es posible en diversas formas. Pasinetti (1985) define la unidad física de capacidad productiva verticalmente integrada para una función de producción "genuina" de cada uno de los bienes del sistema. Con este procedimiento no es posible la consideración de todos los bienes del sistema en unidades físicas homogéneas y, por lo tanto, no es posible una función de producción agregada en unidades físicas homogéneas.

En ciertos casos puede considerarse a las variables en unidades físicas convencionales (toneladas, hectáreas, horas/hombre) usando las convenciones existentes para las unidades dimensionales en funciones matemáticas aplicadas a la economía; para el caso de las "ecuaciones empíricas”, se acepta que son formulaciones matemáticas que reflejan los valores numéricos de las relaciones entre constantes y/o variables diferentes sin necesidad de tener en cuenta sus unidades. Cf. Frits J. de Jong (1967, pp. 34-63). Este procedimiento podría ser objetado en ecuaciones de carácter teórico y no empírico.

En Firmenich (2015) se plantea la medición de las variables de cantidades de bienes y servicios en unidades físicas de flujos de energía sin que esta magnitud guarde ninguna relación con los precios relativos; los bienes alimenticios para la especie humana se miden en las calorías que proporcionan mediante su digestión; el trabajo humano se mide en las calorías consumidas por el cuerpo humano al realizar trabajo mecánico; la energía aplicada en los procesos productivos se mide directamente en unidades energéticas, los bienes de capital y las manufacturas en general se miden en el costo de producción energético acumulado que les ha dado origen.
} 
2.1.1. El sector agropecuario: produce un bien salario que es exportable. La explotación extensiva de la tierra implica la casi inexistencia de inversión de capital fijo o industrial. El capital invertido es una porción del producto que se reinvierte en el período siguiente, (semilla o ganado reservado para reproducción). La tierra es una variable stock ${ }^{2}$. Puede tenerse tierra ociosa sin coste. La función de producción técnica es:

$$
Y=f\left(T, L_{Y}, Y_{K}\right) ; Y=A \cdot \min \left\{T^{0}, \frac{L_{Y}}{b}, \frac{Y_{K}}{a}\right\}, \quad \frac{Y_{K}}{a} \leq T^{0}, \frac{L_{Y}}{b} \leq T^{0}
$$

$Y$ es el bien primario, $T^{0}$ es la cantidad de tierra fértil disponible, $L_{Y}$ es el trabajo empleado en la producción e $Y_{K}$ es la cantidad del bien primario usada como capital reproductivo. La tecnología tiene coeficientes fijos: $a$ y $b$; $A$ es un coeficiente de productividad.

2.1.2. El sector industrial: su producción es sustitutiva de importaciones de manufacturas livianas de consumo final y no es competitiva internacionalmente; abastece el mercado interno con políticas proteccionistas. Los bienes de capital (máquinas-herramientas e insumos intermedios) son importados. El ahorro doméstico sólo se invierte en construcción y producción de herramientas simples, la inversión significativa es la importación de bienes de capital. La función de producción técnica depende del trabajo y la inversión depende del bien de capital importado, $I_{k}$ :

$$
\frac{\mathrm{dK}}{\mathrm{dt}}=\boldsymbol{I}_{\boldsymbol{K}}
$$

La función de producción técnica de este sector es:

$$
Q=g\left(L_{Q}, K\right)=B \cdot \min \left\{c \cdot L_{Q}, K\right\} \quad, \quad \frac{K}{L_{Q}}=c
$$

$Q$ es el bien manufacturado, $K$ el capital empleado, $c$ una constante, $B$ un coeficiente de productividad y $L_{Q}$ la cantidad de trabajo empleado en producir el bien manufacturado. Dado que el factor escaso son los bienes de capital importado, la función se expresa como:

$$
Q=B \cdot K, \quad K \leq K_{0}
$$

$K_{0}$ es el capital total instalado; la producción puede darse con capacidad instalada ociosa, aunque ello implicará un coste. El sistema tiene cambios estructurales discretos muy espaciados que modifican los valores de $B$ y $c$.

\subsection{La optimización en cada sector productivo.}

2.2.1. En el sector agropecuario: es un oligopolio en el mercado doméstico; considerando la conducta de los terratenientes agremiados como una colusión, asumiremos que tiene una estructura de monopolio. Se asume la hipótesis de país pequeño: la demanda externa del bien primario es infinitamente elástica al precio internacional exógeno.

La maximización de beneficios implica, para el mercado interno, la igualación del ingreso marginal $(I M g)$ del monopolio con su coste marginal $(C M g)$; pero en el mercado externo se puede vender cualquier cantidad al precio internacional dado. Sean: $r$ el beneficio del sector agropecuario como productor monopolista, $p_{y}$ el precio doméstico del bien agropecuario, $Y^{d}$ la cantidad comprada por la demanda interna, $t c^{*}$ el tipo de cambio real, $p_{y}^{*}$ el precio internacional exógeno del bien exportable, $X$ la cantidad del bien primario exportada, $w$ el salario nominal (igual en toda la economía) e $i$ la tasa normal de beneficio del capital (equiparable a la tasa de interés). Así, tenemos:

\footnotetext{
${ }^{2}$ Para que las unidades del factor tierra sean homogéneas con las de las restantes variables físicas, las unidades de superficie de Tierra pueden medirse por la cantidad de energía solar que pueden recibir y aprovechar con la fotosíntesis, de modo que una unidad de superficie puede ser representada por una magnitud en unidades de energía que mide su capacidad productiva agrícola. La misma magnitud de superficie equivaldrá a diferentes magnitudes de energía en función de la fertilidad del suelo (Firmenich 2015).
} 


$$
r=p_{Y} \cdot Y^{d}+\mathrm{tc}^{*} \cdot p_{Y}^{*} \cdot X-w \cdot L_{Y}-(1+i) \cdot p_{Y} \cdot Y_{K} \geq 0
$$

En el mercado interno, considerando el trabajo y el capital proporcionales a la producción vendida internamente resulta (Apéndice Matemático Deducción 1):

$$
\begin{aligned}
& \frac{1}{\varepsilon}=-\frac{Y^{d}}{p_{Y}} \cdot \frac{\mathrm{dp}_{Y}}{\mathrm{dY}^{d}} \quad, \quad \varepsilon>1 \\
& \boldsymbol{p}_{Y}=\frac{\boldsymbol{w} \cdot \boldsymbol{b}}{\boldsymbol{A} \cdot\left(\mathbf{1}-\frac{1}{\varepsilon}\right)-(1+\boldsymbol{i}) \cdot \boldsymbol{a}}
\end{aligned}
$$

$\boldsymbol{\varepsilon}$ es la elasticidad-precio de la demanda interna. El precio interno del bien salario determina la cantidad $Y^{d}$ que se venderá en el mercado doméstico. Para que existan ventas en el mercado interior, debe ser:

$$
\begin{gathered}
\boldsymbol{p}_{\boldsymbol{Y}} \geq \mathrm{tc}^{*} \cdot \boldsymbol{p}_{\boldsymbol{Y}}^{*} \\
\mathrm{tc}{ }^{*}=\mathrm{tc} \cdot \frac{p^{*}}{p}
\end{gathered}
$$

tc es el tipo de cambio nominal, $p^{*} \mathrm{y} p$ son los índices de precios exteriores y domésticos respectivamente. Los beneficios del productor agropecuario en las exportaciones (denotando con el doble apóstrofe " las variables relativas al sector externo), son:

$$
r^{\prime \prime}=\mathrm{tc}^{*} \cdot p_{Y}^{*} \cdot X-w \cdot L_{Y}{ }^{\prime \prime}-(1+i) \cdot \mathrm{tc}^{*} \cdot p_{Y}^{*} \cdot Y_{K}{ }^{\prime \prime} \geq 0 \quad \Rightarrow \mathrm{tc}^{*} \cdot p_{Y}^{*} \cdot\left[X-(1+i) \cdot Y_{K}{ }^{\prime \prime}\right] \geq w \cdot L_{Y}{ }^{\prime \prime}
$$

La renta diferencial de la tierra juega un papel en la variación de las exportaciones ante la variación relativa entre el salario nominal y el tipo de cambio real. Esto puede verse afectado por variaciones exógenas del precio internacional del bien salario exportable.

Si el ingreso por exportaciones genera beneficios, el sector agropecuario producirá el máximo permitido por la tierra disponible, venderá en el mercado interno la cantidad que maximice su beneficio monopólico, ahorrará cierta cantidad para inversión reproductiva y el resto de la producción será vendida en el mercado externo:

$$
X=Y-Y^{d}-Y_{K}
$$

Las variables de la función de producción técnica serán constantes a sus valores máximos iniciales. No necesariamente las variables endógenas son incógnitas, ni las constantes datos conocidos (Pasinetti 1985, p. 88).

La maximización de beneficios del sector determina:

$$
Y=Y^{0}=A \cdot \min \left\{T^{0}, \frac{L_{Y}^{0}}{b}, \frac{Y_{K}^{0}}{a}\right\}, \quad Y_{K}=Y_{K}^{0}=T^{0} \cdot a, \quad L_{Y}=L_{Y}^{0}=T^{0} \cdot b
$$

El supraíndice 0 indica magnitudes constantes al valor físico máximo inicial.

Podemos expresarlo como:

$$
Y^{0}=A \cdot \frac{L_{Y}^{0}}{b}=A \cdot \frac{Y_{K}^{0}}{a}
$$

Los ingresos agropecuarios agregados son la suma de los ingresos de todas las explotaciones. Considerando al sector como un monopolista, los beneficios son:

$$
R=p_{Y} \cdot Y^{d}+\mathrm{tc}^{*} \cdot p_{Y}^{*} \cdot X-w \cdot L_{Y}^{0}-(1+i) \cdot p_{Y} \cdot Y_{K}^{0}
$$

En los beneficios del sector existe la tasa de beneficio normal sobre el capital adelantado más la renta monopólica en el mercado interno. 
2.2.2. En el sector industrial: existen muchas pequeñas y medianas empresas de capital y tecnología similares. La naturaleza no transable de esta producción, sumado a que los industriales no pueden desinvertir para invertir en el sector agropecuario, convierte a la inversión en bienes de capital importado en un activo específico, existiendo costes hundidos: caídas de la demanda interna generan pérdidas en la amortización de la inversión. Los precios no surgen de la optimización de un mercado competitivo. El precio de las manufacturas será superior al competitivo porque existe un aseguramiento empresario (Segura, 1993, p. 57).

Si un productor representativo pudiera maximizar sus beneficios como en un mercado competitivo, siendo $p_{K}^{*}$ el precio del bien de capital importado e $i$ el beneficio normal del capital, el precio de competencia perfecta igual al Coste Total Medio (CTMe), con CMg = CTMe, sería (Apéndice Matemático Deducción 2):

$$
p_{q}=\frac{w+t c^{*} \cdot p_{k}^{*} \cdot c \cdot(1+i)}{B \cdot c} .
$$

La oferta industrial sería infinitamente elástica al precio determinado por el coste, según el salario nominal y el tipo de cambio real, que determina el coste del capital importado. Pero no es así en este caso.

La línea de producción del bien $Q$ con todos los costes, incluyendo la necesidad de aseguramiento es:

$$
p_{q} \cdot Q=w \cdot L_{q}+t c^{*} \cdot p^{*}{ }_{k \cdot} \cdot K \cdot(1+i)+i \cdot t c^{*} \cdot p^{*}{ }_{k \cdot}\left(K_{0}-K\right)=C T .
$$

Cuando $K_{0}=K$ no hay costes adicionales. Pero si el capital empleado es menor al instalado, surge un coste financiero por capacidad instalada ociosa, elevando el CTMe. Aplicando propiedad distributiva y cancelando en la expresión anterior y sustituyendo según la función de producción, es:

$$
C T=p_{q} \cdot Q=\frac{w \cdot Q}{B \cdot c}+t c^{*} \cdot p_{k}^{*} \cdot \frac{Q}{B}+i \cdot t c^{*} \cdot p_{k}^{*} \cdot K_{0}
$$

El CTMe incluyendo el coste por el aseguramiento es:

$$
\text { CTM }=\frac{w}{B \cdot c}+\frac{t c^{*} \cdot p_{k}^{*}}{B}+i \cdot t c^{*} \cdot p_{k}^{*} \cdot \frac{K_{0}}{Q}=\frac{w+t c^{*} \cdot p_{k}^{*} \cdot c \cdot\left(1+i \cdot \frac{K_{0}}{K}\right)}{B \cdot c}
$$

El término constante en la línea de producción no es un Coste Fijo sino un coste irrecuperable. Con la función de beneficios:

$$
\pi=p_{q} \cdot Q-\left[\frac{w \cdot Q}{B \cdot c}+t c^{*} \cdot p_{k}^{*} \cdot \frac{Q}{B}+i \cdot t c^{*} \cdot p_{k}^{*} \cdot K_{0}\right]
$$

la maximización de beneficios para el productor pequeño o mediano, que toma la tasa de interés como dada, implicaría pérdidas, pues el precio resultaría:

$$
\frac{\partial \pi}{\partial Q}=\mathbf{0} \quad p_{q}=\frac{w}{B \cdot c}+t c^{*} \cdot \frac{p_{k}^{*}}{B}
$$

Pero este precio es menor que el de competencia perfecta y menor que CTMe con aseguramiento por los activos específicos:

$$
\frac{w}{B \cdot c}+t c^{*} \cdot \frac{p_{k}^{*}}{B}<\frac{w}{B \cdot c}+t c^{*} \cdot \frac{p_{k}^{*}}{B} \cdot(1+i)<\frac{w}{B \cdot c}+t c^{*} \cdot \frac{p_{k}^{*}}{B} \cdot\left(1+i \cdot \frac{K_{0}}{K}\right)
$$

El precio de venta deberá ser mayor y la cantidad vendida menor que los valores correspondientes al punto de la eficiencia teórica. Aunque esto implica operar en la zona en que el $\mathrm{CMg}$ es menor que el CTMe decreciente, la atomización del sector impide una colusión. Ante una caída de la demanda, las empresas pequeñas y medianas que puedan se contentarán con cubrir el CTMe y las más apremiadas 
aceptarán vender transitoriamente por encima de su CMg pero por debajo de su CTMe.

En general resultará:

$$
\frac{w}{B \cdot c}+t c^{*} \cdot \frac{p_{k .}^{*}}{B} \cdot(1+i) \leq p_{q} \leq \frac{w}{B \cdot c}+t c^{*} \cdot \frac{p_{k .}^{*}}{B} \cdot\left(1+i \cdot \frac{K_{0}}{K}\right)
$$

El precio de los bienes manufacturados incluirá entonces un "sobreprecio" pero sin cuasirrentas; el "exceso" de precio disminuirá a medida que se incrementa el uso del capital instalado hasta su pleno empleo. Si $K=K_{0}$, el precio resulta igual al de competencia perfecta.

Si hay capacidad instalada ociosa, será $C M g<p_{q}<C T M e$; habrá pérdidas que desaparecerán si el precio es de competencia perfecta. Los capitalistas industriales necesitan la expansión del mercado interno hasta que no exista capacidad instalada ociosa. Supondremos, salvo que se aclare lo contrario, que $p_{q}=C T M e:$

$$
p_{q}=\frac{w+t c^{*} \cdot p_{k}^{*} \cdot c \cdot\left(1+i \cdot \frac{K_{0}}{K}\right)}{B \cdot c}
$$

Los beneficios agregados de los industriales son (Apéndice Matemático Deducción 3):

$$
\Pi=i . t c^{*} \cdot p^{*} \cdot\left[2 \cdot K-K_{0}\right]
$$

\subsection{Las preferencias de los consumidores.}

Se supone que todos los individuos tienen iguales preferencias con diferentes restricciones presupuestarias ${ }^{3}$. Las preferencias son lexicográficas: primero, se satisface las Necesidades Básicas de alimentación; después, se satisface las Necesidades de Status con bienes de industria nacional; finalmente se consume bienes de lujo importados.

Si $Y$ es el bien alimenticio, $Q$ es el bien manufacturado nacional e $I_{c}$, es el bien de consumo de lujo importado, una combinación de bienes cualquiera $A_{1}=\left(Y_{1}, Q_{1}, I_{c}{ }_{1}\right)$, será preferida a otra combinación, $A_{i}=\left(Y_{i}, Q_{i}, I_{c i}\right)$, si se cumple:

$\left.1^{\circ}\right) Y_{1}>Y_{i} / Y_{1} \leq Y^{\mathrm{NBS}}$

$\left.2^{\circ}\right) Y_{1}=Y_{i}, Q_{1}>Q_{i} / Q_{1} \leq Q^{\text {NSS }}$

$\left.3^{\circ}\right) Y_{1}=Y_{i}, Q_{1}=Q_{i}, I_{c 1}>I_{c i}$.

NBS y NSS indican el valor de las variables para Necesidades Básicas Satisfechas y Necesidades de Status Satisfechas. Cuando los ingresos aumentan cualquier individuo prefiere consumir bienes "más sofisticados"4. El nivel de renta diferencia tres estratos sociales:

a) el sector de bajos ingresos, asalariado

b) el sector de ingresos medios, pequeños y medianos capitalistas

c) el sector de altos ingresos, terratenientes latifundistas.

\footnotetext{
${ }^{3}$ Cf. Musgrave, R. A. y Musgrave, P. B. (1992, p. 69) "...sostener que las necesidades se experimentan individualmente no supone negar la existencia de la interacción social. ... Además, la proposición de que las necesidades se experimentan individualmente no excluye el altruismo. ... Sin embargo, aceptando todo esto, lo que importa aquí es que la satisfacción se experimenta en último término por A y B de forma individual, y no por una tercera entidad misteriosa denominada $A+B^{\prime \prime}$.

${ }^{4}$ Pasinetti (1985, p. 82) fundamenta esta conducta en la evidencia empírica de la Ley de Engel y sostiene que la utilidad marginal de los bienes preferidos en primer orden llega a ser negativa con los aumentos de renta. En rigor, para que se pase a una cesta con igual cantidad del bien preferido en primer orden y mayor cantidad del bien preferido en el orden siguiente, la condición necesaria y suficiente es que la utilidad marginal del primero sea inferior a la del segundo, lo que efectivamente ocurre cuando se alcanza el nivel de consumo de "necesidad básica satisfecha", que no es necesariamente idéntico a la saciedad.
} 
Las preferencias lexicográficas no pueden ser representadas por una función de utilidad (Henderson \& Quandt, 1991, p. 15) ${ }^{5}$ lo que implica que no se puede deducir funciones de demanda con maximización restringida de la utilidad.

\subsection{Función de consumo, restricciones presupuestarias de los consumidores y funciones de demanda.}

2.4.1. Función de consumo y restricción presupuestaria. Partimos de una simplificación de la teoría del consumo y del ahorro basada en la renta del ciclo vital (Dornbusch, Fischer \& Startz, 2002, pp. 315318), incorporamos en las expectativas de ahorro y desahorro los períodos de edades activa y pasiva y los períodos sin ingresos por actividades productivas en edad activa; se define una restricción presupuestaria general que varía según la situación particular de ingresos.

Los ingresos pueden ser salariales, por beneficios empresariales, por renta de la tierra o cualquier combinación de ingresos. Los ingresos monetarios corrientes de actividades productivas, $P$, son:

$$
P=w+\pi+r
$$

La función de consumo general de cada individuo en unidades monetarias es:

$$
C=u \cdot W R+v \cdot P=p_{y} \cdot Y^{d}+p_{q} \cdot Q^{d}+t c^{*} \cdot p^{*}{ }_{y} \cdot I_{c}{ }^{d}
$$

$C$ es el consumo, $W R$ es la riqueza poseída al margen de los ingresos de actividades productivas, $u$ y $v$ son propensiones marginales a consumir.

La riqueza inicial puede tener cualquier valor, incluso 0 , pero todos acumulan riqueza con el ahorro por actividades productivas. Es decir: $\quad W R(t)=W R_{0}+\int(1-v) \cdot P(t) \cdot d t$

Los individuos pueden cambiar de clase social cambiando de categoría de renta. Si los ingresos merman, los individuos se financian con desahorro.

La relación entre restricción presupuestaria y consumo es:
$u \cdot\left[0+\int(1-v) \cdot P(t) \cdot d t\right]+v \cdot[P<w]$
$u \cdot\left[0+\int(1-v) \cdot P(t) \cdot d t\right]+v \cdot[P=w]$
$\Rightarrow C=p_{y} \cdot Y^{d}$
$\Rightarrow C=p_{y \cdot} Y^{d}+p_{q} \cdot Q^{d}$
$Y^{d} \leq Y^{N B S}$
$u \cdot\left[W R_{0}+\int(1-v) \cdot P(t) \cdot d t\right]+v \cdot[P=w+\pi]$
$\Rightarrow C=p_{y \cdot} Y^{d}+p_{q} \cdot Q^{d}$
$Y^{d}=Y^{N B S}, Q^{d}<Q^{N S S}$
$u \cdot\left[W R_{0}+\int(l-v) \cdot P(t) \cdot d t\right]+v \cdot[P=w+\pi+r]$
$\Rightarrow C=p_{y \cdot} Y^{d}+p_{q} \cdot Q^{d}+t c^{*} \cdot p^{*}{ }_{\text {Ic }} \cdot I_{C}{ }^{d}$
$Y^{d}=Y^{N B S}, Q^{d} \leq Q^{N S S}$
$Y^{d}=Y^{N B S}, Q^{d}=Q^{N S S}$

El primer nivel de renta corresponde a asalariados en situación de desempleo o subempleo. El segundo formaliza a los asalariados empleados. El tercer caso corresponde a los industriales pequeños y medianos. El cuarto, representa a los terratenientes.

El precio internacional del bien de lujo importado, $p_{\text {Ic }}^{*}$, es exógeno.

\subsubsection{Las funciones de demanda.}

Para bienes normales, dada la renta, la cantidad demandada depende del precio en relación inversa y, según la Ley de Engel, para un incremento de la renta la demanda aumenta en proporciones decrecientes. Según Pasinetti (1985, pp. 83-84), para incrementos sucesivos de renta, la demanda de un bien se incrementa en proporciones decrecientes hasta independizarse de la renta y, para niveles suficientemente elevados de renta, también del precio. No necesitamos suponer esto último. Es suficiente aceptar la modificación en los escalones lexicográficos de consumo según los niveles de renta; en todos los casos la cantidad demandada dependerá inversamente del precio para una renta constante.

\footnotetext{
${ }^{5}$ Sen (1976, p. 53) comenta la misma implicación formal en cuanto a la imposibilidad de representar una ordenación lexicográfica completa por una función real, pero referido a la función de bienestar social y generaliza la afirmación en la Nota 7 de p. 121: "Con una ordenación lexicográfica, las alternativas pueden estar completamente ordenadas sin que haya una escala de utilidad (ni siquiera ordinal) que pueda ser ajustada a ella".
} 
La demanda de mercado de cada bien es la suma horizontal, para cada nivel de la renta nacional, de las demandas de los tres niveles de renta per cápita; los supraíndices 1, 2 y 3 indican los niveles de asalariados, capitalistas y terratenientes. Cada demanda es un múltiplo del individuo representativo.

2.4.2.1. Demanda de mercado del bien primario en el mercado interno.

$$
Y^{d}=h^{1}\left(p_{y}, W\right)+h^{2}\left(p_{y}\right)+h^{3}\left(p_{y}\right) \Rightarrow \begin{gathered}
Y^{d}=h\left(p_{y}, W\right) \\
\frac{\partial h}{\partial p_{y}}<0, \frac{\partial h}{\partial W}>0
\end{gathered}
$$

$W$ representa la masa salarial en la renta nacional: $\quad W=w \cdot\left(L_{q}+L_{y}{ }^{0}\right)$

La demanda de alimentos aumenta con incrementos de renta de los asalariados porque existen Necesidades Básicas insatisfechas en desempleados y subempleados.

2.4.2.2. Demanda de mercado del bien manufacturado no transable.

$$
\begin{array}{r}
Q^{d}=j^{1}\left(p_{q}, W\right)+j^{2}\left(p_{q}, \Pi\right)+j^{3}\left(p_{q}\right) \Rightarrow Q^{d}=j\left(p_{q}, W, \Pi\right) \\
\frac{\partial j}{\partial p_{q}}<0, \frac{\partial j}{\partial W}>0, \frac{\partial j}{\partial \Pi}>0
\end{array}
$$

$\Pi$ es la sumatoria de los beneficios de los capitalistas industriales. La demanda se expande con incremento en $W$ y/o en $\Pi$ porque existen en ambos sectores Necesidades de Status insatisfechas.

\subsubsection{Demanda del bien de lujo importado.}

Tenemos:

$$
I_{c}^{d}=k\left(p_{I c}^{*}\right), \frac{\partial k}{\partial p_{I c}^{*}}<0
$$

El aumento del tipo de cambio no afecta la demanda del bien de lujo importado porque incrementa en igual proporción el precio en moneda doméstica y los ingresos de los exportadores-consumidores. Siendo este precio exógeno y no pudiendo, como regla dominante, los industriales convertirse en terratenientes, consideramos constante la demanda del bien de lujo importado:

$$
I_{c}{ }^{d}=I_{c}{ }^{0}
$$

\section{5. La definición del equilibrio.}

2.5.1. El mercado de dinero. Existe una oferta de dinero real pasivo (Olivera, 1968) que se adapta a la demanda de saldos reales según se incrementen los precios (Olivera, 1960). Los precios son inflexibles a la baja, bajando más lentamente que lo que suben (Olivera, 1984). Por simplicidad supondremos que los precios no bajan. El numerario es una unidad monetaria abstracta de dinero fiduciario. Es decir, hay precios absolutos en unidades monetarias abstractas (precios relativos al dinero pasivo fiduciario) (Olivera, 1986). La ecuación del mercado de dinero resulta suprimida junto con la definición del numerario fiduciario, pero oferta y demanda de dinero real están en equilibrio siempre y la dinámica inflacionaria es (Apéndice Matemático Deducción 4):

$$
\frac{d p}{d t}=l\left(\frac{d w}{d t}, \frac{d t c}{d t}, p^{*}\right) \quad, \quad l^{\prime}>0 \quad, \quad \frac{d w}{d t}, \frac{d t c}{d t} \geq 0
$$

2.5.2. EI mercado de bienes del sector agropecuario: existe siempre equilibrio de cantidades. Cualquier cantidad ofrecida se distribuye entre un mercado interno monopólico y un mercado externo que absorbe 


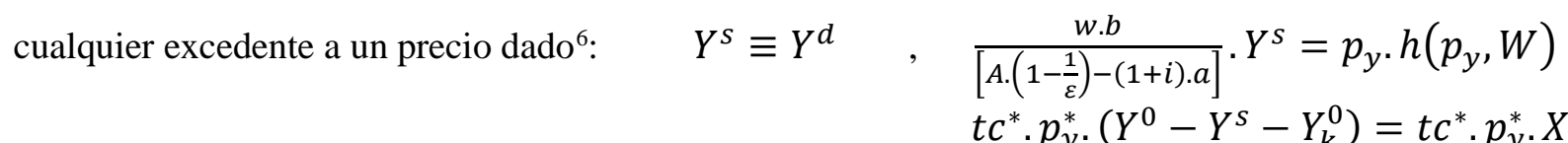

$Y^{S}$ es la cantidad vendida por el monopolio en el mercado interno idéntica a la cantidad comprada. Esto ya estaba descrito por la ecuación: $\quad X=Y-Y^{d}-Y_{k}$

2.5.3. EI mercado de bienes del sector manufacturero: existe equilibrio de cantidades. La oferta, cuya elasticidad-precio tiende a infinito, Ec. (10), enfrenta una demanda de pendiente negativa para una renta dada:

$$
Q^{s}=Q^{d} \quad ; \quad\left[\frac{w}{B . c}+t c^{*} \cdot \frac{p_{k}^{*}}{B}+i \cdot t c^{*} \cdot \frac{p_{k}^{*}}{B} \cdot \frac{K_{0}}{K}\right] \cdot B \cdot K=p_{q} \cdot j\left(p_{q}, W, \Pi\right)
$$

La demanda de capital es una demanda derivada de la demanda del bien final:

$$
K=\frac{1}{B} \cdot j\left(p_{q}, W, \Pi\right)
$$

2.5.4. EI mercado de trabajo: se supone que la oferta crece a la misma tasa constante que la población:

$$
L^{S}=L_{0 .} e^{n . t}
$$

La demanda agregada de trabajo es la sumatoria de la demanda constante en el sector agropecuario y la demanda variable del sector manufacturero, que es una demanda derivada de la demanda del bien manufacturado:

$$
\begin{gathered}
L^{d}=L_{q}+L_{y}^{0} \quad, \quad L_{y}^{0}=b \cdot T^{0}, \quad L_{q}=\frac{Q^{s}}{B . c} \\
\Rightarrow L_{q}=\frac{1}{B . c} \cdot j\left(p_{q}, W, \Pi\right)
\end{gathered}
$$

El sistema no garantiza que se vacíe el mercado de trabajo. Es posible el desempleo por escasa demanda ante el crecimiento de la oferta:

$$
\text { w. }\left(L^{S}-L^{d}\right) \geq 0
$$

Los desempleados financian su consumo, con desahorro:

$$
w \cdot\left(L^{S}-L^{d}\right) \geq u \cdot \int[(1-v) \cdot P(t) \cdot d t]+v \cdot[P<w]
$$

2.5.5. El sector externo: no se garantiza el equilibrio de la balanza comercial entre importaciones y exportaciones. La variable Déficit Externo, $D E$, representa un exceso de demanda de divisas financiado con endeudamiento externo. Existe Inversión Extranjera Directa, IED*, como variable exógena en términos netos de flujo de capital; puede ser positiva, nula o negativa:

$$
D E=-\left[p^{*} \cdot X+I E D^{*}-p^{*}{ }_{I c} \cdot I_{c}{ }^{d}-p^{*}{ }_{k} \cdot I_{k}\right]
$$

\subsubsection{El ahorro y la inversión.}

La única inversión capaz de dinamizar el crecimiento y crear empleo es la de bienes de capital importado; así, el ahorro relevante es el ahorro nacional de divisas, $S$, que sólo puede ser ahorrado por los terratenientes exportadores:

$$
S=p^{*} \cdot X-p^{*}{ }_{I c} \cdot I_{c}{ }^{d}
$$

\footnotetext{
${ }^{6}$ Dada la formalización adoptada, un salario muy elevado junto con un tipo de cambio real suficientemente bajo determinarían una reducción de la cantidad ofrecida, sin costes por dejar tierras ociosas, manteniéndose aproximadamente iguales las ventas en el mercado interno monopólico y reduciéndose las exportaciones. En esto jugaría un rol importante la renta diferencial de la tierra. Las explotaciones agropecuarias cuyos rendimientos no permitan satisfacer la desigualdad $t c^{*} \cdot p^{*} \cdot\left[X-(1+i) . Y_{k}\right.$ "] $\geq w \cdot L_{y}$ ” dejarán de producir para la exportación. Si el mercado interno monopólico no absorbe las cantidades que se dejan de exportar, se reducirá la oferta global.
} 
La ecuación del mercado de divisas se puede representar como la inversión en bienes de capital importados igualada a la sumatoria de ahorro nacional de divisas y flujos externos de divisas:

$$
\text { tc. } p^{*}{ }_{k} \cdot d K / d t=t c \cdot\left[S+I E D^{*}+D E\right]
$$

\subsubsection{El equilibrio entre oferta agregada y demanda agregada.}

El valor agregado por cada sector implica restar de las ventas el valor de los inputs intermedios. En el sector agropecuario el input es el bien primario invertido para reproducción; en el sector industrial los únicos imputs intermedios son los incluidos en los bienes de capital importados, de modo que son incluidos en la misma variable que la amortización del capital. Al no formalizar el sector público no existen impuestos indirectos y el Producto Nacional Neto $(P N N)$ resulta igual a la renta nacional:

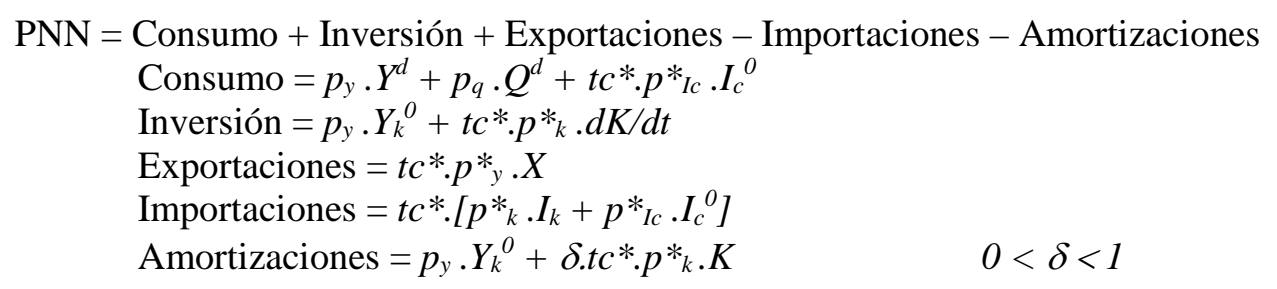

El parámetro $\delta$ representa el desgaste de máquinas y herramientas más los inputs intermedios importados. Sustituyendo y cancelando es:

$$
P N N=p_{y} \cdot Y^{d}+p_{q} \cdot Q^{d}+t c^{*} \cdot p^{*} \cdot X-t c^{*} \cdot p^{*}{ }_{k} \cdot K
$$

Como condición de equilibrio, la suma de los ingresos netos de la renta de la tierra, de los beneficios industriales y de la masa salarial, debe igualar al $P N N$ en unidades monetarias:

$$
R+\Pi+W=P N N
$$

\subsection{Ecuaciones, variables y parámetros del modelo.}

El modelo tiene las 26 ecuaciones numeradas, 27 variables endógenas $\left(Y, L_{y}, Y_{k}, K, I_{k}, Q, L_{q}, \boldsymbol{\varepsilon}, Y^{d}, p_{y}\right.$, $\left.w, i, t c^{*}, t c, p, X, R, P_{q}, \Pi, W, Q^{d}, I_{c}{ }^{d}, L^{S}, L^{d}, D E, S, P N N\right), 8$ variables exógenas $\left(T^{0}, p^{*}, p^{*}, p^{*}{ }_{k}, K_{0}\right.$, $\left.p^{*}{ }_{\text {Ic }}, L_{0}, I E D^{*}\right)$ y 7 parámetros $(A, a, b, B, c, n, \delta)$.

Existe un grado de libertad. Siguiendo a Pasinetti (1985, pp. 141-143), el sistema de precios desempeña dos funciones: una, referida a las decisiones descentralizadas en donde los precios proveen una evaluación de los inputs para la producción; la otra referida a la distribución de la renta, donde salario y tasa de beneficios son variables distributivas. El modelo tiene tres precios endógenos: los precios de los dos bienes producidos y el precio de la divisa extranjera. Además, están las dos variables distributivas.

Los precios no son independientes de la distribución de la renta (Sraffa, 1983). Alguna variable distributiva deberá ser determinada exógenamente. Una decisión de política económica fija una variable, (no necesariamente el salario nominal o la tasa de beneficios), determinando la distribución de la renta $y$, con ello, todas las variables endógenas.

En este modelo, la escasez de divisas está en el centro del conflicto distributivo; es un juego de suma cero en relación al ahorro en divisas. Las decisiones institucionales sobre la política de ingresos pueden beneficiar a los terratenientes exportadores, (incluyendo períodos en que los capitalistas industriales aceptan políticas recesivas para recomponer la balanza de pagos y disminuir los salarios reales), o a los capitalistas industriales y los asalariados, vinculados al mercado interno.

Se analizarán los efectos que tiene sobre la evolución del sistema y del conflicto distributivo una 
política económica que, según las alianzas sociales, gestiona la variación de los precios relativos mediante la variación del tipo de cambio real.

\section{Efectos distributivos de las variaciones del tipo de cambio real.}

El efecto de las variaciones del tipo de cambio real sobre la distribución del ingreso se analiza con un sistema de 5 ecuaciones simultáneas con 5 incógnitas.

Las variables incógnitas y las ecuaciones son (Apéndice Matemático Deducción 5):

$$
\frac{\partial R}{\partial t c^{*}}, \frac{\partial \Pi}{\partial t c^{*}}, \frac{\partial W}{\partial t c^{*}}, \frac{\partial j}{\partial t c^{*}}, \frac{\partial p_{q}}{\partial t c^{*}}
$$

(3.III.) $\frac{\partial W}{\partial t c^{*}}=\frac{w}{B . c} \cdot \frac{\partial j}{\partial t c^{*}}$

(3.IV.) $\frac{\partial j}{\partial t c^{*}}=\frac{\partial j}{\partial p_{q}} \cdot \frac{\partial p_{q}}{\partial t c^{*}}+\frac{\partial j}{\partial W} \cdot \frac{\partial W}{\partial t c^{*}}+\frac{\partial J}{\partial \Pi} \cdot \frac{\partial \Pi}{\partial t c^{*}}$

(3.V.) $\quad \frac{\partial p_{q}}{\partial t c^{*}}=\frac{p_{k}^{*}}{B} \cdot\left[1+i \cdot \frac{K_{0}}{K} \cdot\left(1-\frac{t c^{*}}{j_{\left(p_{q}, W, \Pi\right)}} \cdot \frac{\partial j}{\partial t c^{*}}\right)\right]$

$\Rightarrow \frac{\partial p_{q}}{\partial t c^{*}}=\frac{p_{k}^{*}}{B} \cdot\left[1+i \cdot \frac{K_{0}}{K} \cdot\left(1-\varepsilon_{\left(Q^{d}, t c^{*}\right)}\right)\right]$

3.1. Efectos de la variación del tipo de cambio real sobre los beneficios de los terratenientes.

De la ecuación (3.I.) surge que un aumento del tipo de cambio real mejora sus beneficios:

$$
\frac{\partial R}{\partial t c^{*}}=p_{y}^{*} \cdot\left[y^{0}-Y_{k}^{0} \cdot(2+i)\right]>0
$$

\subsection{Efectos de la variación del tipo de cambio real sobre la cantidad demandada del bien} manufacturado.

Reemplazando (3.V), (3.II) y (3.III) en (3.IV) obtenemos (Apéndice Matemático Deducción 6):

$$
\frac{\partial j}{\partial t c^{*}}=\frac{\frac{\partial J}{\partial p_{q}} \cdot \frac{p_{k}^{*}}{B} \cdot\left(1+i \cdot \frac{K_{0}}{K}\right)+\frac{\partial j}{\partial \Pi} \cdot i \cdot p_{k}^{*} \cdot\left(2 \cdot K-K_{0}\right)}{1+i \cdot \frac{K_{0}}{K} \cdot \frac{t c^{*}}{B} \cdot K \cdot \frac{\partial j}{\partial p_{q}} \cdot \frac{p_{k}^{*}}{B}-\frac{\partial j}{\partial W} \cdot \frac{w}{B \cdot c}-\frac{\partial j}{\partial \Pi} \cdot\left\{i \cdot p_{k}^{*}\left(2 \cdot K-K_{0}\right)+i \cdot t c^{*} \cdot p_{k}^{*} \cdot \frac{2}{B}\right\}}
$$

En el denominador todos los términos son negativos excepto el primero: 1. Dadas las magnitudes involucradas, el denominador es negativo. En el numerador el primer término es negativo. Pero el segundo término será positivo mientras el capital empleado no deje una capacidad ociosa suficientemente grande como para convertirlo en nulo, negativo o muy pequeño; aunque si esto ocurriera, podría variar también el signo del denominador, con lo que la derivada sería negativa.

Si $K=K_{0}$, el denominador es negativo mientas que en el numerador disminuye el valor absoluto del primer término negativo y aumenta el valor absoluto del segundo término positivo. Puede esperarse entonces que el numerador sea positivo y que la derivada sea negativa. Para ello debe cumplirse que:

$$
\begin{gathered}
\frac{\partial j}{\partial p_{q}} \cdot \frac{p_{k}^{*}}{B} \cdot(1+i)>-\frac{\partial J}{\partial \Pi} \cdot i \cdot p_{k}^{*} \cdot K_{0} \\
\Rightarrow \frac{1}{B \cdot K_{0}} \cdot \frac{\partial j}{\partial p_{q}}>-\frac{\partial j}{\partial \Pi} \cdot \frac{i}{(1+i)} \\
\quad \Rightarrow \varepsilon_{\left(Q^{d}, p_{q}\right)}<\frac{\partial j}{\partial \Pi} \cdot p_{q} \cdot \frac{i}{(1+i)}
\end{gathered}
$$


Dada la magnitud numérica de $p_{q}$ es razonable esperar que esta relación se cumpla, aunque depende de comprobaciones empíricas. Aceptando que esta relación se verifica, con $K=K_{0}$, un incremento de $t c^{*}$ hará caer la demanda del bien manufacturado y resultará: $\partial j / \partial t c^{*}<0$

\subsection{Efectos de la variación del tipo de cambio real sobre el precio del bien manufacturado.}

La ecuación (3.V.) permite decir que si la derivada de la demanda del bien manufacturado respecto del tipo de cambio real resulta negativa o nula, el efecto sobre el precio del bien manufacturado será positivo:

$$
\text { (3.V.) } \begin{aligned}
\frac{\partial p_{q}}{\partial t c^{*}} & =\frac{p_{k}^{*}}{B} \cdot\left[1+i \cdot \frac{K_{0}}{K} \cdot\left(1-\frac{t c^{*}}{j_{\left(p_{q}, W, \Pi\right)}} \cdot \frac{\partial j}{\partial t c^{*}}\right)\right] \\
& \Rightarrow \frac{\partial j}{\partial t c^{*}} \leq 0 \Rightarrow \frac{\partial p_{q}}{\partial t c^{*}}>0
\end{aligned}
$$

Es importante destacar que aun si la derivada de la demanda del bien manufacturado, $j$, respecto de $t c^{*}$ fuera positiva, el efecto sobre el precio seguiría siendo positivo dentro de ciertos límites que conviene estudiar.

En efecto, teniendo en cuenta que Ec. (3.V.) es:

$$
\frac{\partial p_{q}}{\partial t c^{*}}=\frac{p_{k}^{*}}{B} \cdot\left[1+i \cdot \frac{K_{0}}{K} \cdot\left(1-\varepsilon_{\left(Q^{d}, t c^{*}\right)}\right)\right]
$$

para que el efecto de una subida del tipo de cambio real sobre el precio del bien manufacturado fuera negativo sería necesario que la elasticidad de la demanda respecto al tipo de cambio fuera positiva y bastante mayor que uno; debería cumplirse (Apéndice Matemático Deducción 7):

$$
\varepsilon_{\left(Q^{d}, t c^{*}\right)}>1+\frac{K}{i} \cdot K_{0}
$$

No hay razones ni teóricas ni empíricas para aceptar semejante suposición. Esto se confirma por el hecho de que si tomamos la ecuación del precio, Ec. (10), simplificada para la situación $K=K_{0}$, resulta:

$$
p_{q}=\frac{\left[w+(1+i) \cdot t c^{*} \cdot p_{k}^{*} \cdot c\right]}{B \cdot c} \Rightarrow \frac{\partial p_{q}}{\partial t c^{*}}=(1+i) \cdot p_{k}^{*} \cdot \frac{c}{B \cdot c}>0
$$

Así, podemos aceptar que $\partial p_{q} / \partial t c^{*}>0$. Esta conclusión refuerza la plausibilidad de la conclusión sobre el efecto negativo de la subida del tipo de cambio real sobre la demanda del bien manufacturado y, por lo tanto, cabe descartar la hipótesis de que la elasticidad de esta demanda respecto al tipo de cambio pueda ser positiva. En efecto:

$$
\begin{aligned}
& \text { (3.IV.) } \quad \frac{\partial j}{\partial t c^{*}}=\frac{\partial j}{\partial p_{q}} \cdot \frac{\partial p_{q}}{\partial t c^{*}}+\frac{\partial j}{\partial W} \cdot \frac{\partial W}{\partial t c^{*}}+\frac{\partial J}{\partial \Pi} \cdot \frac{\partial \Pi}{\partial t c^{*}} \\
& \frac{\partial j}{\partial p_{q}}<0, \frac{\partial p_{q}}{\partial t c^{*}}>0, \frac{\partial j}{\partial W}>0, \frac{\partial W}{\partial t c^{*}}<0, \frac{\partial J}{\partial \Pi}>0, \frac{\partial \Pi}{\partial t c^{*}}<0 \\
& \Rightarrow \frac{\partial j}{\partial t c^{*}}<0 \\
& \frac{\partial j}{\partial t c^{*}}<0 \Rightarrow \varepsilon_{\left(Q^{d}, t c^{*}\right)}=\frac{t c^{*}}{j_{\left(p_{q}, W, \Pi\right)}} \cdot \frac{\partial J}{\partial t c^{*}}<0
\end{aligned}
$$




\subsection{Efectos de la variación del tipo de cambio real sobre los beneficios industriales.}

Retomando la discusión sobre el efecto de un aumento de $t c^{*}$ sobre los beneficios de los industriales, teníamos:

$$
\begin{aligned}
\frac{\partial \Pi}{\partial t c^{*}} & =i \cdot p_{k}^{*} \cdot\left[2 \cdot K-K_{0}\right]+i \cdot \frac{t c^{*}}{B} \cdot p_{k}^{*} \cdot 2 \cdot \frac{\partial j}{\partial t c^{*}} \\
\Rightarrow \frac{\partial \Pi}{\partial t c^{*}} & =i \cdot p_{k}^{*} \cdot\left[2 \cdot K-K_{0}\right]+i \cdot p_{k}^{*} \cdot 2 \cdot K \cdot \frac{t c^{*}}{Q^{d}} \cdot \frac{\partial j}{\partial t c^{*}}
\end{aligned}
$$

Si la derivada de la demanda del bien manufacturado respecto al tipo de cambio real es negativa, siendo $K=K_{0}$, para que toda la expresión sea negativa debe cumplirse:

$$
\text { 2. } K_{0} \cdot \frac{t c^{*}}{Q^{d}} \cdot \frac{\partial j}{\partial t c^{*}}<-K_{0} \quad \Rightarrow \varepsilon_{\left(Q^{d}, t c^{*}\right)}<-\frac{1}{2}
$$

Si esto se cumple, entonces:

$$
\frac{\partial \Pi}{\partial t c^{*}}=i \cdot p_{k}^{*} \cdot K_{0} \cdot\left[1+2 \cdot \varepsilon_{\left(Q^{d}, t c^{*}\right)}\right]<0
$$

El impacto de una subida de $t c^{*}$ en los ingresos de los industriales dependerá de si existe capacidad productiva ociosa o no y de la elasticidad de la demanda respecto a $t c^{*}$.

Hemos considerado por simplicidad que $p_{q}=C T M e$. Pero esto sólo es cierto para $K=K_{0}$; en caso contrario, la caída de la demanda interna hará que $C M g<p_{q}<C T M e$ y la reducción de beneficios podrá convertirse en pérdidas:

$$
\frac{\partial \Pi}{\partial t c^{*}}=i \cdot p_{k}^{*} \cdot\left[2 \cdot K \cdot\left(1+\varepsilon_{\left(Q^{d}, t c^{*}\right)}\right)-K_{0}\right]<0
$$

La exigencia sobre el valor absoluto de la elasticidad se relaja en esta expresión. Dependiendo de la diferencia entre $K$ y $K_{0}$, un valor absoluto de $\varepsilon<1 / 2$ puede convertir en negativa la expresión. Cuanto mayor sea la capacidad instalada ociosa, menor será el valor absoluto de elasticidad requerido para producir una caída en los beneficios industriales.

Podemos afirmar plausiblemente que, con desempleo de capital instalado, la caída de la demanda originada en la subida del tipo de cambio real eleva el precio del bien manufacturado, pero lo ubica por debajo del CTMe; así, la subida del tipo de cambio real genera una caída de los beneficios industriales.

Con pleno empleo de capital y trabajo, la situación depende de las elasticidades. En casos de sobreempleo laboral, las consecuencias de una subida del tipo de cambio real pueden ser cuando menos neutras sobre los beneficios del sector industrial y quizás inclusive positivas. La razón es que, si bien una subida del tipo de cambio real reduce las cantidades de equilibrio, también eleva el precio de venta y reduce el empleo laboral reduciendo el salario real, pero sin generar capacidad productiva ociosa significativa del capital instalado. El efecto final sobre los beneficios depende de la elasticidad-precio de la demanda.

Esto daría origen a unas alianzas oscilantes del sector industrial. En épocas de recesión y alto desempleo de capital y trabajo, su alianza sería con los asalariados para lograr un aumento de la demanda interna mediante aumentos de salarios nominales trasladados a los precios de las manufacturas, mientras se contiene el tipo de cambio nominal; en consecuencia, cae el tipo de cambio real. Pero en épocas de pleno empleo, la tendencia es la subida del salario real que reduce el beneficio empresarial, a la vez que aumenta el consumo del bien salario reduciendo los saldos exportables y aumenta la importación de inputs intermedios. En este caso la alianza del sector se volcaría hacia los terratenientes; el objetivo sería devaluar el tipo de cambio nominal congelando los salarios nominales, produciendo una subida del tipo de cambio real. Así se mejoraría la balanza de pagos mientras se incrementan los beneficios 
agropecuarios y se reduce el salario real con un efecto neutro o positivo sobre los beneficios industriales.

\subsection{Efectos de la variación del tipo de cambio real sobre los ingresos de los asalariados.}

Sobre la situación de los asalariados, teníamos:

$$
\begin{gathered}
\frac{\partial W}{\partial t c^{*}}=\frac{w}{B . c} \cdot \frac{\partial j}{\partial t c^{*}} . \\
\frac{\partial j}{\partial t c^{*}}<0 \quad \Rightarrow \frac{\partial W}{\partial t c^{*}}<0
\end{gathered}
$$

Entonces:

Surge así el conflicto distributivo ante la crisis de la balanza de pagos.

\section{Efectos de la variación del tipo de cambio real sobre la renta nacional en el corto plazo.}

\subsection{Efectos de la variación del tipo de cambio real sobre el producto físico.}

Partiendo de la ecuación (25), tras breves manipulaciones, se obtiene el $P N N$ en unidades físicas (Apéndice Matemático Deducción 8). Su variación es:

$$
\frac{\partial P N N(F)}{\partial t c^{*}}=\left(1-\frac{\delta}{B}\right) \cdot \frac{\partial j\left[p_{q}, W, \Pi\right]}{\partial t c^{*}} .
$$

Interesa el signo de esta expresión. Hemos aceptado que la derivada de la demanda de consumo del bien manufacturado respecto a $t c^{*}$ es negativa pero $(1-\delta / \mathrm{B})>0$; en consecuencia, una subida de tc* hará caer el $P N N$ en términos físicos, contradiciendo el concepto habitual de que una depreciación real de la moneda incrementa el producto "real".

Un alza de $t c^{*}$ reduciría el producto físico porque el producto agropecuario no cambia y cae la demanda (igual a la producción) del bien manufacturado nacional debido al aumento de su precio.

\subsection{Efectos de la variación del tipo de cambio real sobre el producto medido en unidades monetarias.}

Según la ecuación (25), con la simplificación $p_{y}=t c^{*} \cdot p^{*}$ y y realizando algunas sustituciones resulta (Apéndice Matemático Deducción 9):

$$
\frac{\partial P N N}{\partial t c^{*}}=p_{y}^{*} \cdot\left(Y^{0}-Y_{k}^{0}\right)+\left[\frac{\partial p_{q}}{\partial t c^{*}}-\delta \cdot \frac{p_{k}^{*}}{B}\right] \cdot Q^{d}+\left(p_{q}-\delta \cdot t c^{*} \cdot \frac{p_{k}^{*}}{B}\right) \cdot \frac{\partial j}{\partial t c^{*}}
$$

El primer término es positivo y su valor absoluto elevado por la magnitud de la diferencia entre el producto agropecuario y los inputs reproductivos. El segundo término es positivo y una idea sobre la magnitud de su valor absoluto lo da la cantidad demandada del bien manufacturado. En el tercer término, la expresión entre paréntesis es positiva, pero su magnitud es comparativamente pequeña, pues es menor que el precio del bien manufacturado; la derivada de la demanda del bien manufacturado respecto de tc* puede ser negativa, pero difícilmente su valor absoluto convierta en negativo todo el miembro derecho. Es muy probable que la derivada del $P N N$ en unidades monetarias respecto a $t c^{*}$ sea positiva. La diferencia entre el $P N N$ en términos físicos y su medición en términos "reales" con el habitual índice deflactor, es cualitativa. La deflación del PNN podría hacer creer que el producto neto "real" ha crecido cuando el producto neto físico ha descendido. 


\subsection{Efecto de un incremento del tipo de cambio real sobre la inflación.}

Una subida de $t c *$ resulta inflacionaria, por sus efectos sobre los precios domésticos de ambos bienes y por el encarecimiento en moneda doméstica del bien de lujo importado:

Esto es lo que refleja la ecuación (16):

$$
\begin{gathered}
p_{y} \geq t c^{*} \cdot p_{y}^{*} \Rightarrow \frac{\partial p_{y}}{\partial t c^{*}} \geq 0 \\
p_{q}=\frac{w+t c^{*} \cdot p_{k}^{*} \cdot c \cdot\left(1+i \frac{K_{0}}{K}\right)}{\frac{\partial\left(t c^{*} \cdot p_{I c}^{*}\right)}{\partial t c^{*}}>0} \quad \Rightarrow \frac{\partial p_{q}}{\partial t c^{*}}>0
\end{gathered}
$$

$$
\frac{d p}{d t}=l\left(\frac{d w}{d t}, \frac{d t c}{d t}, p^{*}\right), \quad l^{\prime}>0 \quad, \quad \frac{d w}{d t}, \frac{d t c}{d t} \geq 0
$$

Es posible la existencia de inflación importada. Dado que $t c^{*}=t c . p^{*} / p$, un aumento de $p^{*}$ implicará un aumento del tipo de cambio real, con los mismos efecto sobre los precios domésticos y sobre la distribución de la renta. Tal es el caso de los shocks externos.

El mismo fenómeno se produce ante un deterioro de los términos de intercambio. El deterioro de precios relativos entre materias primas exportadas y bienes de capital importados tiene como primera manifestación el estrangulamiento de la balanza de pagos. Pero además produce un alza del tipo de cambio real con consecuencias inflacionarias y de redistribución de la renta nacional a favor del sector exportador, junto a una caída del producto nacional neto en términos físicos.

\section{Efectos de la variación del tipo de cambio real en la dinámica del crecimiento económico.}

\subsection{La dinámica general del crecimiento.}

Partiendo de la ecuación (25), con la simplificación $p_{y}=t c^{*} \cdot p^{*}$ y suponiendo $K=K_{0}$, obtenemos la expresión general de la dinámica del crecimiento (Apéndice Matemático Deducción 10):

$$
\begin{aligned}
\frac{d P N N}{d t}=\frac{d w}{d t} & \frac{K}{c}+\frac{w}{c} \cdot \frac{d K}{d t}+p_{k}^{*} \cdot t c^{*}\left\{K \frac{d i}{d t}+(1+i-\delta) \frac{d K}{d t}\right\} \\
& +\left\{p_{y}^{*}\left(Y^{0}-Y_{k}^{0}\right)+(1+i-\delta) p_{k}^{*} \cdot K\right\} \frac{d t c^{*}}{d t}
\end{aligned}
$$

\subsection{La dinámica del crecimiento equilibrado.}

En la ecuación de la dinámica del crecimiento del $P N N$ están las variables nominales, $d w / d t$ y $d i / d t$, cuyo crecimiento -en forma independiente del crecimiento de las variables físicas y haciendo abstracción de la distribución de la renta-, sólo implicaría un crecimiento inflacionario del PNN; está la variable representativa del crecimiento económico real: $d K / d t$; también está la variable que refleja el conflicto distributivo y los eventuales shocks externos, como el shock petrolero o el deterioro de los términos de intercambio: $d t c^{*} / d t$.

Hemos deducido la ecuación del crecimiento suponiendo $K=K_{0}$. Para estudiar un crecimiento equilibrado corresponde incluir pleno empleo laboral y ausencia de inflación; esto implica suponer que el conflicto distributivo se encuentra en un estado de paz social y, por lo tanto, no desencadena la inflación de costes. El pleno empleo laboral implica: $w \cdot\left(L^{S}-L^{d}\right)=0$. Partiendo de esta condición se obtiene la inversión necesaria en función del crecimiento de la fuerza laboral (Apéndice Matemático Deducción 11): 


$$
\frac{d K}{d t}=c \cdot n \cdot L_{0} \cdot e^{n \cdot t}
$$

Por otra parte, la tasa normal de beneficio, $i$, es el coste de oportunidad representado por la tasa de interés nominal. Considerando constante la tasa de interés real, $i^{*}$, la tasa nominal es:

$$
i^{*}=\frac{(i-\pi)}{(1+\pi)} \quad \Rightarrow i=i^{*}+\left(1+i^{*}\right) . \pi
$$

Si $\pi=0$, la tasa nominal resulta igual a la tasa real y la suponemos constante. En tal caso, el salario nominal constante equivale a un salario real constante. Si no hay una puja distributiva que incentive la variación de precios relativos, entonces $t c^{*}$ también es constante.

En estas condiciones del crecimiento equilibrado será: $d w / d t=0, d i / d t=0, d t c * d t=0$. Sustituyendo estas derivadas y la dinámica de inversión con pleno empleo en la ecuación del crecimiento se obtiene:

$$
\frac{d P N N}{d t}=\left[\frac{w}{c}+p_{k}^{*} \cdot t c^{*} \cdot(1+i-\delta)\right] \cdot c \cdot n \cdot L_{0} \cdot e^{n \cdot t}
$$

Reordenando términos en la expresión entre corchetes y reemplazando por Ec. (10) en condiciones de $K=K_{0}$, resulta la ecuación de la dinámica del crecimiento equilibrado:

$$
\frac{d P N N}{d t}=\left[p_{q} \cdot B-p_{k}^{*} \cdot t c^{*} \cdot \delta\right] \cdot c \cdot n \cdot L_{0} \cdot e^{n \cdot t}
$$

Para valores constantes de estas variables y parámetros, la renta nacional tendrá un crecimiento equilibrado a la tasa de crecimiento de la población. Pero existe un valor de $t c *$ que anula el crecimiento de la renta nacional:

$$
p_{q} \cdot B-p_{k}^{*} \cdot t c^{*} \cdot \delta=0 \quad \Rightarrow t c^{*}=\frac{p_{q \cdot B}}{p^{*} \cdot \delta} \Leftrightarrow \frac{d P N N}{d t}=0
$$

Cuanto mayor sea la productividad tecnológica industrial, $B$, mayor será ese nivel de $t c^{*}$. Lo mismo cabe decir de la disminución de $\delta$, que representa también los inputs importados: una estrategia de sustitución de importaciones, con menor proporción de componentes importados, permitiría un crecimiento equilibrado con un $t c *$ más elevado, haciendo más competitiva internacionalmente la manufactura nacional.

Un incremento de $B$ puede surgir de mejoras tecnológicas al adaptar la tecnología de los bienes de capital importado. Las mejoras de productividad surgen también de mejoras organizativas en la administración y en el proceso productivo, como el caso del just in time ${ }^{7}$.

Un $t c^{*}$ mayor que este valor crítico generaría una caída exponencial de la renta nacional, ya que convertiría en negativa la expresión de la dinámica del crecimiento equilibrado. Este riesgo no es menor cuando se intenta mejorar la balanza de pagos con fuertes devaluaciones/depreciaciones sucesivas que intentan superar la inflación en contextos de conflicto distributivo. Este nivel crítico de $t c^{*}$ es muy relevante para el conflicto distributivo y para la restricción de divisas que financian el crecimiento.

\footnotetext{
${ }^{7}$ La existencia de un nivel crítico más alto de $t c^{*}$ no indica necesariamente que sea deseable ubicar la economía en una senda de crecimiento con ese $t c^{*}$ constante más elevado; existirían de todos modos los efectos distributivos e inflacionarios analizados. La eventualidad de que eso fuera posible y deseable exigiría políticas fiscales correctoras de las perturbaciones inflacionarias y distributivas.
} 


\subsection{La restricción del sector externo al crecimiento equilibrado.}

La condición de pleno empleo laboral supone:

$$
\begin{aligned}
& d K / d t=c . n \cdot L_{0} \cdot e^{n . t} \\
& \text { La ecuación (23 bis) es: } \quad \frac{d K}{d t}=\frac{\left[S+I E D^{*}+D E\right]}{p_{k}^{*}} \text {. }
\end{aligned}
$$

Igualando con la ecuación anterior, tomando logaritmos y derivando, resulta:

$$
\frac{\left[\frac{d S}{d t}+\frac{d I E D^{*}}{d t}+\frac{d D E}{d t}\right]}{\left[S+I E D^{*}+D E\right]}=n .
$$

La tasa de crecimiento de la disponibilidad de divisas para financiar el crecimiento equilibrado debe igualar la tasa de crecimiento de la población. Pero cabe prestar atención a las expresiones del numerador. De (10) y (8) resulta:

$$
\frac{d S}{d t}=p_{y}^{*} \cdot \frac{d X}{d t}-p_{I c}^{*} \cdot \frac{d I_{c}^{d}}{d t} \quad, \quad \text { siendo } \quad \frac{d X}{d t}=-\frac{d Y^{d}}{d t}
$$

El aumento del ahorro de divisas exige que disminuya el consumo interno del bien salario, con perjuicio para los asalariados, o que disminuya el consumo del bien de lujo importado, con perjuicio para los terratenientes, o ambas cosas. Existe un conflicto distributivo en torno al incremento de $t c^{*}$ (para aumentar los saldos exportables) y al uso de las divisas disponibles (para importar o bienes de capital o bienes de lujo).

El escenario sería diferente si los saldos exportables pudieran aumentar sin reducir el consumo interno, pero ello exige un aumento continuo de productividad en el sector agropecuario:

$$
Y=A \cdot \min \left\{T^{0}, \frac{L_{y}}{b}, \frac{Y_{k}}{a}\right\} \text {. }
$$

Un incremento del parámetro tecnológico $A$ y una reducción de los parámetros $b$ y $a$, permitiría que $Y$ pudiera crecer superando la situación de la Ec. (1 bis):

$$
Y^{0}=A \cdot \frac{L_{y}^{0}}{b}=A \cdot \frac{Y_{k}^{0}}{a} .
$$

La evidencia empírica en países semiindustrializados exportadores de materias primas sugiere que la financiación del crecimiento basado en importaciones de bienes de capital no ha sido posible por vía de un crecimiento exponencial del ahorro en divisas.

Además, la $I E D^{*}$ es exógena; no cabe esperar su evolución a la tasa de crecimiento de la población. Inclusive, cuando cada ingreso de capital por IED* se convierte en un stock de inversiones que produce manufacturas no transables pero remite utilidades en divisas a una casa matriz extranjera, aparece un componente negativo en esta variable.

La conclusión es general para la estrategia de Industrialización Sustitutiva de Importaciones, basada en $I E D^{*}$, para satisfacer la demanda interna, sin economías escala o con tecnología obsoleta que la hacen no competitiva internacionalmente.

Si, como extremo simplificador, fuera: $d S / d t=0, d\left(I E D^{*}\right) / d t=0$, entonces (por Ec. 23 bis y Deduccón 11) será la deuda externa la que crecerá exponencialmente para financiar el crecimiento equilibrado:

$$
\frac{\frac{d D E}{d t}}{\left[S+I E D^{*}+D E\right]}=n \quad, \quad D E=\frac{\left(p_{k}^{*} \cdot c \cdot n \cdot L_{0}\right)}{\left(S_{0}+I E D_{0}^{*}\right)} \cdot e^{n \cdot t}
$$


Cualquier tasa de crecimiento de la disponibilidad de divisas inferior a la tasa de crecimiento de la oferta de trabajo generará desempleo laboral.

Cabe destacar que los saldos exportables constantes del bien primario no sólo implican tener que financiar la dinámica de la inversión de capital con IED o por endeudamiento externo, sino que, con ese aparente "crecimiento equilibrado" habría una reducción constante del consumo per cápita del bien alimenticio, sería una reducción sostenida del salario real a la tasa de crecimiento de la población.

Esta dinámica no es posible con estabilidad de precios, ya que la masa salarial, con salario nominal constante y pleno empleo, crece a la tasa de crecimiento de la oferta de trabajo; pero esa masa salarial creciente debe comprar una cantidad fija de bienes salarios exportables, con una necesaria tendencia al alza de $p_{y}$.

La restricción tecnológica de la productividad agropecuaria genera inflación y reducción del salario real, incentivando el conflicto distributivo mientras la restricción en la disponibilidad de divisas genera desempleo. En estas condiciones, no existe posibilidad de crecimiento equilibrado.

\subsection{La dinámica del crecimiento en situación de estanflación.}

La ecuación general de la dinámica del crecimiento vista es:

$$
\begin{aligned}
\frac{d P N N}{d t}=\frac{d w}{d t} & \frac{K}{c}+\frac{w}{c} \cdot \frac{d K}{d t}+p_{k}^{*} \cdot t c^{*}\left\{K \frac{d i}{d t}+(1+i-\delta) \frac{d K}{d t}\right\} \\
& +\left\{p_{y}^{*}\left(Y^{0}-Y_{k}^{0}\right)+(1+i-\delta) p_{k}^{*} \cdot K\right\} \frac{d t c^{*}}{d t}
\end{aligned}
$$

Supongamos que se frena el crecimiento de las divisas disponibles porque no aumentan las exportaciones, no se reducen las importaciones, se estanca la $I E D^{*}$ y los acreedores externos restringen el flujo del crédito.

La ecuación del crecimiento supone que $K=K_{0}$, o sea el ingreso de divisas puede financiar las importaciones de inputs intermedios y la reposición del capital instalado; pero no se incrementa la capacidad instalada, de modo que: $d K_{0} / d t=0$.

Recordando la ecuación del PNN en términos físicos y hallando la dinámica del crecimiento en unidades físicas, obtenemos:

$$
P N N(F)=Y^{0}-Y_{K}^{0}+(B-\delta) \cdot K \Rightarrow \frac{d P N N(F)}{d t}=(B-\delta) \cdot \frac{d K}{d t}
$$

Para $d K / d t=0$ resulta $d[P N N(F)] / d t=0$. Es decir que, anulando en la ecuación general del crecimiento los términos en que $d K / d t$ aparece como factor, la ecuación reducida del crecimiento expresaría un proceso puramente inflacionario, en que el crecimiento de la renta nacional sólo ocurre en unidades monetarias, pero con crecimiento cero del producto físico:

$$
\frac{d P N N}{d t}=\frac{d w}{d t} \cdot \frac{K}{c}+p_{k}^{*} \cdot t c^{*} \cdot K \cdot \frac{d i}{d t}+\left\{p_{y}^{*} \cdot\left(Y^{0}-Y_{k}^{0}\right)+(1+i-\delta) \cdot p_{k}^{*} \cdot K\right\} \cdot \frac{d t c^{*}}{d t}
$$

Reemplazando $K=c . L_{0} \cdot e^{\text {n.t }}$ (no existiendo el crecimiento equilibrado, $K$ no crece pero $L$ sí), el crecimiento de los valores nominales de la renta nacional seguiría una trayectoria exponencial a la tasa de crecimiento de la población, rumbo a la hiperinflación. 


\subsection{La dinámica del crecimiento bajo la aplicación del enfoque monetario de la balanza de pagos en situación de estanflación y estrangulamiento del sector externo.}

Supongamos que en este contexto el objetivo de la política económica es contener la inflación y mejorar el saldo de la balanza de pagos para pagar compromisos de deuda externa. Supondremos que el objetivo es una reducción de la tasa de inflación a niveles bajos y constantes -con una tasa de inflación esperada $\pi^{e}$ definida políticamente-, a la vez que lograr saldos favorables en la balanza de pagos para pagar compromisos con acreedores externos.

Para reducir la inflación y el consumo de bienes salario exportables se congela $w$ y se evita la reducción del tipo de interés real $i *$ sosteniendo su valor positivo constante, es decir: $i>\pi$. Al mismo tiempo, para mejorar el saldo de la balanza de pagos, se buscará elevar $t c^{*}$, devaluando $t c$ a una tasa mayor que $\pi$.

Dado que en la estanflación existe estancamiento del producto físico, el objetivo de la tasa de inflación se puede medir por la tasa de crecimiento de la renta nacional en unidades monetarias. Así, los objetivos de esta política económica se pueden formalizar como:

$$
\begin{gathered}
\frac{\frac{d P N N}{d t}}{P N N}=\frac{\frac{d p}{d t}}{p}=\pi \leq \pi^{e} \quad \Rightarrow \frac{d P N N}{d t}=P N N \cdot \pi \leq \pi^{e} \\
\frac{d w}{d t}=0 \quad, \quad \frac{d t c^{*}}{d t}>0 \\
i>\pi \quad, \quad i^{*}=\frac{i-\pi^{e}}{1+\pi^{e}} \quad \Rightarrow i=i^{*}+\left(1+i^{*}\right) \cdot \pi^{e} \quad \Rightarrow \frac{d i}{d t}=\left(1+i^{*}\right) \cdot \frac{d \pi^{e}}{d t}
\end{gathered}
$$

Así, el gobierno actuará para que la ecuación reducida de la dinámica del crecimiento sea:

$$
\text { PNN. } \pi=p_{k}^{*} \cdot t c^{*} \cdot K \cdot\left(1+i^{*}\right) \cdot \frac{d \pi^{e}}{d t}+\left[p_{y}^{*} \cdot\left(Y^{0}-Y_{k}^{0}\right)+(1+i+\delta) \cdot p_{k}^{*} \cdot K\right] \cdot \frac{d t c^{*}}{d t}
$$

Con sucesivas transformaciones desde la ecuación anterior, resulta el tipo de cambio real necesario para los objetivos del ajuste según el enfoque monetario de la balanza de pagos (Apéndice Matemático Deducción 12):

$$
t c^{*}=\frac{\left[-w \cdot L_{0} \cdot e^{n \cdot t} \cdot \pi\right]}{\left[p_{y}^{*} \cdot\left(Y^{0}-Y_{k}^{0}\right)+(1+i-\delta) \cdot p_{k}^{*} \cdot c \cdot L_{0} \cdot e^{n \cdot t}\right] \cdot\left[2 \cdot \pi-\frac{\frac{d t c}{d t}}{t c}\right]-p_{k}^{*} \cdot\left(1+i^{*}\right) \cdot \frac{d \pi^{e}}{d t} \cdot c \cdot L_{0} \cdot e^{n \cdot t}}
$$

Esta expresión tiene dos problemas: según los valores de variables y parámetros, puede ser indeterminada (por anulación del denominador) y podría ser negativa (absurdo) si el denominador fuera positivo. El problema no se resuelve con una tasa de inflación negativa, haciendo positivo el numerador, ya que entonces el denominador sería negativo. Ambos problemas se sortearían si la tasa de inflación no se acelerara, $d \pi / d t=0$, y si la tasa de devaluación/depreciación no se acelerara. En tal caso, una tasa de devaluación igual al doble de la tasa de inflación generaría un valor límite positivo y definido de $t c^{*}$ para $t \rightarrow \infty$ (Apéndice Matemático Demostración 1).

Pero estas suposiciones son contrarias a realidades como la hiperinflación, o la sobrerreacción del tipo de cambio ${ }^{8}$, o los Bancos Centrales que agotan sus reservas ante una fuga de capitales.

Si suponemos que tanto $p$ como tc admiten múltiples derivadas sucesivas positivas respecto al tiempo, entonces, ante una inflación que se acelera a una tasa exponencial, los objetivos planteados de la política de ajuste pueden ser formalizados como funciones exponenciales a algunas tasas particulares:

$$
\frac{d \pi}{d t}=e^{P . t} \quad, \quad \frac{d t c}{d t}=e^{T C . t} \quad, \quad \frac{d i}{d t}=\left(1+i^{*}\right) \cdot \frac{d \pi^{e}}{d t}=\left(1+i^{*}\right) \cdot e^{\Pi \cdot t}
$$

\footnotetext{
${ }^{8}$ Cf. Dornbusch (1976, pp. 1161-1176).
} 


$$
T C>P \quad, \quad \Pi>P
$$

Sustituyendo estas expresiones en la ecuación anterior, el $t c^{*}$ necesario para el éxito del ajuste con el enfoque monetario de la balanza de pagos resulta (Apéndice Matemático Deducción 13):

$$
t c^{*}=\frac{-w}{p_{y}^{*}\left(Y^{0}-Y_{k}^{0}\right) L_{0}^{-1}\left(2 e^{-n . t}-e^{(T C-P-n) \cdot t}\right)+(1+i-\delta) p_{k}^{*} \cdot c\left(2-e^{(T C-P) \cdot t}\right)-p_{k}^{*}\left(1+i^{*}\right) e^{(\Pi-P) \cdot t} \cdot c}
$$

El valor límite con $t \rightarrow \infty$ depende de las relaciones entre las tasas exponenciales.

Suponiendo las condiciones descritas, en el corto plazo el valor de $t c^{*}$ resulta indeterminado, su evolución es inestable y errática con tendencia descendente: su aumento tras cada devaluación se diluye en la dinámica inflacionaria, obligando a sucesivas devaluaciones y subidas del tipo de interés. El resultado es un círculo vicioso que acelera la inflación, la depreciación y el aumento de la tasa de interés nominal, con continua caída del salario real y del tipo de cambio real, rumbo a la hiperinflación.

Para el largo plazo, un valor límite del $t c^{*}$ con $t \rightarrow \infty$ resulta negativo o nulo, lo que económicamente es absurdo (Apéndice Matemático Demostración 2). Este absurdo debe ser interpretado como la imposibilidad de alcanzar el nivel del tipo de cambio real necesario para cumplir el objetivo de esta política económica.

\section{Conclusiones.}

La formalización no neoclásica del modelo colabora para un desempeño más realista del mismo. El sistema posee un grado de libertad. Su equilibrio no existe en el marco de la economía pura, sino que una política económica no neutral gestiona un equilibrio (entendido como determinación del sistema de precios y no como igualación a cero de todos los excesos de demanda) basado en cierta direccionalidad en la distribución de la renta. Esto explica un conflicto distributivo, en un juego de suma cero, en que se lucha por el poder de decidir los valores de $t c$, de $w$ o de $i$, para alcanzar un valor favorable del $t c^{*}$.

En el modelo se integran ecuaciones microeconómicas con ecuaciones y dinámica macroeconómica. Pero la macroeconomía no es la suma de elementos microeconómicos; existe una estructura sistémica que trasciende la economía pura, representada por la existencia de un grado de libertad.

Los valores de algunas variables endógenas pueden ser constantes.

Las funciones de demanda no se deducen de funciones de utilidad; surgen de preferencias lexicográficas sobre Necesidades Básicas de subsistencia y Necesidades de Status, combinadas con la ley de Engel y aceptando el ahorro y el desahorro.

El estudio del crecimiento económico combinado con el conflicto distributivo puede abordarse mejor al diferenciar variables físicas de variables monetarias. Para ello, se supera el concepto de "variables reales" considerando la producción en términos técnicos y unidades físicas como algo independiente de los valores monetarios.

Esto permite analizar la estanflación considerando el estancamiento de las variables físicas junto al crecimiento de las variables monetarias.

El modelo puede tener interés teórico para cualquier economía agroexportadora semiindustrializada. Si bien algunas economías agropecuarias no exportan bienes salario, el coste de oportunidad de uso de la tierra plantea un dilema entre dedicarla a la producción de bienes salario o a la producción de bienes exportables. 
La dinámica del modelo evidencia los cuellos de botella que estrangulan el desarrollo en la estructura descripta. En estas condiciones estructurales no es posible el crecimiento equilibrado y la dinámica genera un conflicto distributivo que tiene en su centro la escasez de divisas. Queda en evidencia un problema económico estructural con diversas facetas:

a) Hay una contradicción entre el aprovechamiento de la capacidad exportadora del sector agropecuario y el salario real de los trabajadores urbanos porque se exporta "bienes salario". Dada la diferencia de poder de mercado de los exportadores entre el mercado interno y el internacional, el precio doméstico resulta mayor o igual al precio internacional.

b) Hay una contradicción entre un tipo de cambio real alto para exportar y un salario real alto para sostener con el consumo el crecimiento del mercado interno. El tipo de cambio real alto implica un salario real bajo y viceversa.

c) Hay una contradicción entre el crecimiento de la renta per cápita en base al desarrollo industrial para el mercado interno y el financiamiento de dicho desarrollo mediante las divisas generadas por la renta de la tierra. Si los terratenientes exportadores no invierten en la industria y pueden tener tierra ociosa con coste 0 se plantea un conflicto por el uso de las divisas generadas con mayor poder en manos de terratenientes.

d) Hay una contradicción entre crecimiento económico sostenido en base a una producción industrial para el mercado interno (no transable) y la balanza de pagos, que se estrangula por una alta "propensión marginal a importar" debido a la dependencia tecnológica de la industria, al aumento del consumo de los bienes salario que reduce los saldos exportables y a la falta de incentivos para los agroexportadores bajo la hegemonía industrialista.

La gestión macroeconómica sólo a través de la política de ingresos es incapaz de resolver el problema estructural y genera ciclos de expansión y recesión. El crecimiento equilibrado exige cambios estructurales que permitan que:

a) la inversión en $I+D+I$ permita una producción industrial competitiva internacionalmente sin un tipo de cambio real artificialmente alto;

b) no existan costes hundidos en la industria, lo que exige diversificación industrial, la posibilidad de transferir inversiones entre el sector agropecuario y el industrial y el desarrollo del sector servicios con capacidad exportadora;

c) la innovación tecnológica en el sector agropecuario permita incrementar la producción de bienes salario a la tasa de crecimiento de la población para sostener el salario real sin reducir los saldos exportables;

d) el desarrollo del capital humano permita un salario real alto sin un tipo de cambio real artificialmente bajo, para que resulte financiable el consumo de los bienes exportables al precio internacional en moneda nacional;

e) sea imposible la tenencia sin costo fiscal de tierra productiva ociosa, reduciendo el poder de mercado sobre el precio de los bienes-salario.

\section{Referencias}

Braun, O., \& Leonard J. (1981). Un modelo de estancamiento económico. Estudio de caso sobre la economía argentina, Desarrollo Económico. Revista de Ciencias Sociales, 80(20), 585-604.

Díaz Alejandro, C.F. (1965). Exchange-rate devaluation in a semi-industrialized country: the experience of Argentina 1955 - 1961, The M.I.T. Press.

Díaz Alejandro, C.F. (1975). Ensayos sobre la historia económica argentina. Buenos Aires: Amorrortu Editores.

Dornbusch, R. (1976). Expectations and Exchange Rate Dynamics. Journal of Political Economy, 84(6), 1161-1176. 
Dornbusch, R., Fischer, S., \& Startz, R. (2002). Macroeconomía, 8ª Ed. Madrid: Mc Graw Hill.

Firmenich, M.E. (2015). Teoría de los sistemas político - económicos, EDUCO, Neuquén.

Frits, J. de Jong (1967). Dimensional analysis for economists. Amsterdam: North - Holland Publishing Company.

Guerberoff, S. (1977). Un análisis de la performance del segmento industrial estable y su impacto en el modelo de crecimiento argentino (1949-1967). Desarrollo Económico. Revista de Ciencias Sociales, 64(16), IDES, enero-marzo.

Henderson, J.M., \& Quandt, R.E. (1991). Teoría microeconómica - Edición revisada y puesta al día. Barcelona: Ariel Economíax.

Musgrave, R.A., \& Musgrave, P.B. (1992). Hacienda pública teórica y aplicada - Quinta edición. Madrid: McGraw Hill.

Olivera, J.H. (1960). La Teoría no Monetaria de la Inflación. El Trimestre Económico, (octubre diciembre), 616 - 628.

Olivera, J.H. (1968). El Dinero Pasivo. El Trimestre Económico, vol. 35, No 140(4) (octubre diciembre), 695 - 706.

Olivera, J.H. (1984). Sur l'inflexibilité des prix a la baise. Revue d'Economie Politique, 6.

Olivera, J.H. (1986). El Patrón monetario. En: Banco Central de la República Argentina, El Banco Central en su $50^{\circ}$ aniversario: 1935-1985.

Pasinetti, L.L. (1985). Cambio estructural y crecimiento económico. Madrid: Ed. Pirámide.

Porto, A. (1975). Un modelo simple sobre el comportamiento macroeconómico argentino en el corto plazo. Desarrollo Económico. Revista de Ciencias Sociales, 59 (15), IDES

Segura, J. (1993). Teoría de le Economía Industrial. Madrid: Editorial Civitas.

Sen, A.K. (1976). Elección colectiva y bienestar social. Madrid: Alianza Universidad.

Sraffa, P. (1983). Producción de Mercancías por Medio de Mercancías. Barcelona: Oikos-tau. 


\section{APÉNDICE MATEMÁTICO}

\section{Deducción 1 \\ Deducción de la elasticidad-precio del bien salario y de su precio monopolista en el mercado interno: ecuaciones (4) y (5)}

Los beneficios del productor agropecuario son:

$$
\begin{gathered}
r=I T-C T \\
r=p_{y} \cdot Y^{d}+t c^{*} \cdot p_{y}^{*} \cdot X-\left[w \cdot L_{y}+(1+i) \cdot p_{y} \cdot Y_{k}\right] \geq 0
\end{gathered}
$$

En el mercado interno (denotando con el apóstrofe ' las variables relativas al sector interno), considerando las cantidades de trabajo y capital proporcionales a la producción vendida internamente, resulta:

$$
r^{\prime}=p_{y} \cdot Y^{d}-\left[w \cdot L_{y}{ }^{\prime}+(1+i) \cdot p_{y} \cdot Y_{k}{ }^{\prime}\right]
$$

Las relaciones establecidas en la función de producción técnica son:

$$
L_{y}{ }^{\prime}=b \cdot T^{\prime}=b \cdot \frac{Y^{d}}{A} \quad, Y_{k}{ }^{\prime}=a \cdot T^{\prime}=a \cdot \frac{Y^{d}}{A}
$$

donde $a, b$, y A son los parámetros ya definidos. Por lo tanto, reemplazando según estas relaciones resulta:

$$
r^{\prime}=p_{y} \cdot Y^{d}-\left[w \cdot \frac{b}{A} \cdot Y^{d}+(1+i) \cdot p_{y} \cdot \frac{a}{A} \cdot Y^{d}\right]
$$

La maximización de beneficios del monopolista significa:

$$
\begin{gathered}
I M g=C M g \\
\Rightarrow p_{y} \cdot\left(1-\frac{1}{\varepsilon}\right)=w \cdot \frac{b}{A}+(1+i) \cdot p_{y} \cdot \frac{a}{A}
\end{gathered}
$$

$\varepsilon$ es la elasticidad-precio de la demanda interna, por lo tanto, su inversa es:

$$
\frac{1}{\varepsilon}=-\left(\frac{Y^{d}}{p_{y}}\right) \cdot \frac{d p_{y}}{d Y^{d}} \quad, \quad \varepsilon>1
$$

De la expresión de igualdad de $I M g$ y $C M g$ surgen el precio interno del bien salario y la cantidad $Y^{d}$ que se venderá en el mercado interno:

$$
\begin{gathered}
p_{y} \cdot\left(1-\frac{1}{\varepsilon}\right)=w \cdot \frac{b}{A}+(1+i) \cdot p_{y} \cdot \frac{a}{A} \Rightarrow p_{y} \cdot\left(1-\frac{1}{\varepsilon}\right) \cdot A=w \cdot b+(1+i) \cdot a \cdot p_{y} \\
\Rightarrow\left[\left(1-\frac{1}{\varepsilon}\right) \cdot A-(1+i) \cdot a\right] \cdot p_{y}=w \cdot b \\
p_{y}=\frac{w \cdot b}{\left(1-\frac{1}{\varepsilon}\right) \cdot A-(1+i) \cdot a}
\end{gathered}
$$

\section{Deducción 2}

Deducción de la maximización de beneficios del sector industrial en competencia perfecta

Si un hipotético productor representativo maximizara sus beneficios del modo habitual para un mercado competitivo, teniendo en cuenta las relaciones estipuladas en la función de producción técnica, denotando al precio del bien de capital importado como $p_{k}^{*} \mathrm{y}$ al beneficio normal del capital equiparable a la tasa de interés $i$, resultaría:

$$
\pi=p_{q} \cdot Q-\left[w \cdot L_{q}+t c^{*} \cdot p_{k}^{*} \cdot K \cdot(1+i)\right] \geq 0
$$

Las relaciones establecidas en la función de producción técnica son:

$$
K=\frac{Q}{B} \quad, \quad L_{q}=\frac{K}{c}=\frac{Q}{B . c}
$$


Reemplazando estas relaciones en la función de beneficios resulta:

$$
\begin{gathered}
\pi=p_{q} \cdot Q-\left[w \cdot \frac{Q}{B \cdot c}+t c^{*} \cdot p_{k}^{*} \cdot \frac{Q}{B} \cdot(1+i)\right] \geq 0 \\
\frac{\partial \pi}{\partial Q}=0 \Rightarrow p_{q}=\frac{w}{B \cdot c}+t c^{*} \cdot p_{k}^{*} \cdot \frac{(1+i)}{B}
\end{gathered}
$$

Deducción 3

Deducción de los beneficios agregados de los capitalistas industriales: ecuación (11)

El Costo Total de cada productor industrial es la sumatoria de los costos laborales, los costos de capital empleado como input y el costo financiero de la capacidad instalada:

$$
C T=w \cdot L_{q}+t c^{*} \cdot p_{k}^{*} \cdot K+i \cdot t c^{*} \cdot p_{k}^{*} \cdot K_{0}
$$

Según la función de producción técnica:

Sustituyendo en $C T$ resulta:

$$
Q=B . K \Rightarrow K=\frac{Q}{B} \quad, \quad \frac{K}{L_{q}}=c \Rightarrow L_{q}=\frac{K}{c}
$$

El Costo Total Medio resulta:

$$
\begin{aligned}
& C T=w \cdot \frac{K}{c}+t c^{*} \cdot p_{k}^{*} \cdot \frac{Q}{B}+i \cdot t c^{*} \cdot p_{k}^{*} \cdot K_{0} \\
& C T=w \cdot \frac{Q}{B \cdot c}+t c^{*} \cdot p_{k}^{*} \cdot \frac{Q}{B}+i \cdot t c^{*} \cdot p_{k}^{*} \cdot K_{0}
\end{aligned}
$$

$$
\begin{gathered}
C T M e=\frac{C T}{Q}=\frac{\left[w \cdot \frac{Q}{B \cdot c}+t c^{*} \cdot p_{k}^{*} \cdot \frac{Q}{B}+i \cdot t c^{*} \cdot p_{k}^{*} \cdot K_{0}\right]}{Q}=\frac{w}{B \cdot c}+t c^{*} \cdot \frac{p_{k}^{*}}{B}+i \cdot t c^{*} \cdot p_{k}^{*} \cdot \frac{K_{0}}{B \cdot K} \\
C T M e=\left[w+t c^{*} \cdot p_{k}^{*} \cdot c+i \cdot t c^{*} \cdot p_{k}^{*} \cdot c \cdot \frac{K_{0}}{K}\right] \cdot \frac{1}{B \cdot c}
\end{gathered}
$$

Por lo tanto, si el precio es igual al CTMe resulta:

$$
p_{q}=\left[w+t c^{*} \cdot p_{k}^{*} \cdot c+i \cdot t c^{*} \cdot p_{k}^{*} \cdot c \cdot \frac{K_{0}}{K}\right] \cdot \frac{1}{B \cdot c}
$$

Con un precio que cubre el costo unitario de producción los beneficios económicos de un productor representativo serán normales o nulos. Un beneficio económico nulo significa la existencia de un beneficio contable igual al costo de oportunidad de los recursos empleados; dicho costo de oportunidad está representado por los intereses que devengaría el valor monetario del capital empleado, es decir: i. $t c^{*} . p_{k}^{*}$. $K$. El beneficio contable agregado de todos los productores es la participación del capital en la renta nacional.

Los beneficios agregados de todos los capitalistas industriales en la renta nacional serán:

$$
\begin{gathered}
\Pi=\sum_{j=1}^{n}\left[i \cdot t c^{*} \cdot p_{k^{*}}^{*} \cdot K^{j}-i \cdot t c^{*} \cdot p_{k}^{*} \cdot\left(K_{0}^{j}-K^{j}\right)\right] \\
\Pi=\sum_{j=1}^{n} 2 \cdot i \cdot t c^{*} \cdot p_{k}^{*} \cdot K^{j}-i \cdot t c^{*} \cdot p_{k}^{*} \cdot K_{0}^{j} \\
\Pi=i \cdot t c^{*} \cdot p_{k}^{*} \cdot \sum_{j=1}^{n}\left[2 \cdot K^{j}-K_{0}^{j}\right] \\
\Pi=i \cdot t c^{*} \cdot p_{k}^{*} \cdot\left[2 \cdot K-K_{0}\right]
\end{gathered}
$$


Deducción 4

Deducción de la ecuación (16)

En este modelo de 4 bienes, los 2 precios de producción nacional dependen del salario y del tipo de cambio real, en tanto que los 2 precios importados son exógenos y deben ser convertidos a moneda doméstica con el tipo de cambio nominal. Si se define el índice de precios $p=\frac{\sum p_{i}^{t} \cdot q_{i}^{0}}{\sum p_{i}^{0} \cdot q_{i}^{0}}$, en nuestro modelo de cuatro bienes, dos producidos nacionalmente y dos importados, resulta:

$$
p=\frac{p_{Y}^{t} \cdot q_{Y}^{0}+p_{q}^{t} \cdot q_{q}^{0}+p_{I c}^{*} \cdot q_{I c}^{0} \cdot t c+p_{k}^{*} \cdot q_{k}^{0} \cdot t c}{\sum p_{i}^{0} \cdot q_{i}^{0}}
$$

Con excepción de los precios corrientes de los dos bienes de producción doméstica y del tipo de cambio nominal, todas las demás variables son valores fijos. Por lo tanto, podemos expresar el índice de precios como:

$$
p=\alpha_{y} \cdot p_{y}\left(w, t c^{*}\right)+\alpha_{q} \cdot p_{q}\left(w, t c^{*}\right)+\left[\alpha_{k} \cdot p_{k}^{*}+\alpha_{c} \cdot p_{I c}^{*}\right] . t c .
$$

Cada coeficiente $\alpha_{i}=\frac{q_{i}^{0}}{\sum p_{i}^{0} \cdot q_{i}^{0}}$ es constante.

Derivando esta expresión de $p$ con respecto al tiempo resulta:

$$
\frac{d p}{d t}=\alpha_{y} \cdot\left[\frac{\partial p_{y}}{\partial w} \cdot \frac{d w}{d t}+\frac{\partial p_{y}}{\partial t c^{*}} \cdot \frac{d t c^{*}}{d t}\right]+\alpha_{q} \cdot\left[\frac{\partial p_{q}}{\partial w} \cdot \frac{d w}{d t}+\frac{\partial p_{q}}{\partial t c^{*}} \cdot \frac{d t c^{*}}{d t}\right]+\left(\alpha_{k} p_{k}^{*}+\alpha_{c} p_{I c}^{*}\right) \cdot \frac{d t c}{d t}
$$

La derivada del tipo de cambio real con respecto al tiempo es:

$$
\frac{d t c^{*}}{d t}=\frac{p^{*}}{p} \cdot \frac{d t c}{d t}-t c \cdot \frac{p^{*}}{p^{2}} \cdot \frac{d p}{d t} .
$$

Sustituyendo esta derivada del tipo de cambio real, en la derivada del índice de precios con respecto al tiempo y despejando $d p / d t$, se obtiene una ecuación diferencial que depende de las variables $d w / d t, d t c / d t$ y $p^{*}$. Esto es lo que se formula en general en la ecuación (16):

$$
\frac{d p}{d t}=l\left(\frac{d w}{d t}, \frac{d t c}{d t}, p^{*}\right)
$$

\section{Deducción 5}

Deducción de los efectos distributivos de las variaciones del tipo de cambio real

El análisis del efecto de las variaciones del tipo de cambio real sobre la distribución del ingreso nos plantea un sistema de cinco ecuaciones simultáneas con cinco incógnitas, que podemos resolver con sucesivas sustituciones. En efecto, a partir de las ecuaciones:

$$
\begin{gathered}
R=p_{Y} \cdot Y^{d}+\mathrm{tc}^{*} \cdot p_{Y}^{*} \cdot X-w \cdot L_{Y}^{0}-(1+i) \cdot p_{Y} \cdot Y_{K}^{0} \\
\Pi=i \cdot t c^{*} \cdot p_{k}^{*} \cdot\left[2 \cdot K-K_{0}\right] \\
W=w \cdot\left(L_{q}+L_{y}{ }^{0}\right)
\end{gathered}
$$

Sustituyendo, en la ecuación (9), la simplificación $p_{y}=t c^{*} \cdot p^{*}$ y, luego, reemplazando $Y^{d}$ de acuerdo a la ecuación (8) $Y^{0}-Y_{k}^{0}=Y^{d}+X$, resulta:

$$
\begin{aligned}
& R=t c^{*} \cdot p_{y}^{*} \cdot\left[Y^{0}-Y_{k}^{0}-(1+i) \cdot Y_{k}^{0}\right]-w \cdot L_{y}^{0} \\
& \quad \Rightarrow R=t c^{*} \cdot p_{y}^{*} \cdot\left[Y^{0}-Y_{k}^{0} \cdot(2+i)\right]-w \cdot L_{y}^{0}
\end{aligned}
$$

Sustituyendo en la ecuación (11) la variable $K$ según la ecuación (18) $K=\frac{1}{B} \cdot j\left(p_{q}, W, \Pi\right)$, resulta:

$$
\Pi=\left(i . t c^{*} \cdot p_{k}^{*}\right) \cdot\left[\frac{2}{B} \cdot j\left(p_{q}, W, \Pi\right)-K_{0}\right]
$$


Sustituyendo en la ecuación (13) la variable $L_{q}$ según la ecuación (21) $\boldsymbol{L}_{\boldsymbol{q}}=\frac{\mathbf{1}}{\boldsymbol{B} . \boldsymbol{c}} \cdot \boldsymbol{j}\left(\boldsymbol{p}_{\boldsymbol{q}}, \boldsymbol{W}, \boldsymbol{\Pi}\right)$ resulta:

$$
W=w \cdot\left[\frac{j\left(p_{q}, W, \Pi\right)}{B \cdot c}+L_{y}^{0}\right]
$$

Derivando las expresiones halladas de $R, \Pi$ y $W$ respecto al tipo de cambio real obtenemos el sistema de ecuaciones que queremos resolver:

$$
\begin{aligned}
& \text { (3.I.) } \frac{\partial R}{\partial t c^{*}}=p_{y}^{*} \cdot\left[Y^{0}-Y_{k}^{0} \cdot(2+i)\right] \\
& \text { (3.II.) } \frac{\partial \Pi}{\partial t c^{*}}=i \cdot p_{k}^{*} \cdot\left[2 \cdot K-K_{0}\right]+\frac{i}{B} \cdot t c^{*} \cdot p_{k}^{*} \cdot 2 \cdot \frac{\partial j}{\partial t c^{*}} \\
& \text { (3.III.) } \frac{\partial W}{\partial t c^{*}}=\frac{w}{B \cdot c} \cdot \frac{\partial j}{\partial t c^{*}}
\end{aligned}
$$

Pero además en (3.II.) y (3.III.) tenemos:

$$
\text { (3.IV.) } \frac{\partial j}{\partial t c^{*}}=\frac{\partial j}{\partial p_{q}} \cdot \frac{\partial p_{q}}{\partial t c^{*}}+\frac{\partial j}{\partial W} \cdot \frac{\partial W}{\partial t c^{*}}+\frac{\partial J}{\partial \Pi} \cdot \frac{\partial \Pi}{\partial t c^{*}}
$$

Partiendo de la ecuación (10):

$$
p_{q}=\frac{w+t c^{*} \cdot p_{k}^{*} \cdot c+i \cdot t c^{*} \cdot p_{k}^{*} \cdot c \cdot \frac{K_{0}}{K}}{B \cdot c}=\frac{w}{B \cdot c}+\frac{t c^{*} \cdot p_{k}^{*}}{B}+\frac{i \cdot t c^{*} \cdot p_{k}^{*} \cdot K_{0}}{B \cdot K}
$$

Derivando luego $p_{q}$ con respecto al tipo de cambio real, se obtiene:

$$
\begin{aligned}
\frac{\partial p_{q}}{\partial t c^{*}}=0+\frac{p_{k}^{*}}{B} & +\frac{i \cdot p_{k}^{*} \cdot K_{0} \cdot B \cdot K-i \cdot t c^{*} \cdot p_{k}^{*} \cdot K_{0} \cdot \frac{\partial(B \cdot K)}{\partial t c^{*}}}{\frac{\partial p_{q}}{\partial t c^{*}}} \\
& =\frac{p_{k}^{*}}{B}+\frac{i \cdot p_{k}^{*} \cdot K_{0}}{B \cdot K}-\frac{i \cdot p_{k}^{*} \cdot K_{0}}{B \cdot K} \cdot \frac{t c^{*}}{B \cdot K} \cdot \frac{\partial(B \cdot K)}{\partial t c^{*}}
\end{aligned}
$$

Factoreando, resulta:

$$
\frac{\partial p_{q}}{\partial t c^{*}}=\frac{p_{k}^{*}}{B} \cdot\left[1+\frac{i \cdot K_{0}}{K}-\frac{i \cdot K_{0}}{K} \cdot \frac{t c^{*}}{B \cdot K} \cdot \frac{\partial(B \cdot K)}{\partial t c^{*}}\right]
$$

Sustituyendo ahora con la ecuación (18) $K=\frac{1}{B} \cdot j\left(p_{q}, W, \Pi\right)$ es:

$$
\frac{\partial p_{q}}{\partial t c^{*}}=\frac{p_{k}^{*}}{B} \cdot\left[1+\frac{i \cdot K_{0}}{K}-\frac{i \cdot K_{0}}{K} \cdot \frac{t c^{*}}{j\left(p_{q}, W, \Pi\right)} \cdot \frac{\partial j}{\partial t c^{*}}\right]
$$

Por lo tanto, se obtiene:

$$
\text { (3.V.) } \begin{aligned}
\frac{\partial p_{q}}{\partial t c^{*}}=\frac{p_{k}^{*}}{B} & {\left[1+i \cdot \frac{K_{0}}{K} \cdot\left(1-\frac{t c^{*}}{j\left(p_{q}, W, \Pi\right)} \cdot \frac{\partial j}{\partial t c^{*}}\right)\right] } \\
& \Rightarrow \frac{\partial p_{q}}{\partial t c^{*}}=\frac{p_{k}^{*}}{B} \cdot\left[1+i \cdot \frac{K_{0}}{K} \cdot\left(1-\varepsilon_{\left(Q^{d}, t c^{*}\right)}\right)\right]
\end{aligned}
$$

Los efectos distributivos de las variaciones del tipo de cambio real involucran finalmente estas 5 ecuaciones con 5 incógnitas, que son

$$
\frac{\partial R}{\partial t c^{*}}, \frac{\partial \Pi}{\partial t c^{*}}, \frac{\partial W}{\partial t c^{*}}, \frac{\partial j}{\partial t c^{*}}, \frac{\partial p_{q}}{\partial t c^{*}}
$$


Deducción 6

Deducción de los efectos de la variación del tipo de cambio real sobre la cantidad demandada del bien $\underline{\text { manufacturado }}$

Reemplazando (3.V.) en (3.IV.) tenemos:

$$
\frac{\partial j}{\partial t c^{*}}=\frac{\partial j}{\partial p_{q}} \cdot\left[\frac{p_{k}^{*}}{B} \cdot\left(1+i \cdot \frac{K_{0}}{K}-i \cdot \frac{K_{0}}{K} \cdot \frac{t c^{*}}{B \cdot K} \cdot \frac{\partial j}{\partial t c^{*}}\right)\right]+\frac{\partial j}{\partial W} \cdot \frac{\partial W}{\partial t c^{*}}+\frac{\partial j}{\partial \Pi} \cdot \frac{\partial \Pi}{\partial t c^{*}}
$$

Distribuyendo en el primer término del segundo miembro, pasando al primer miembro el término con derivada de la demanda del bien manufacturado respecto al tipo de cambio real y factoreando en el primer miembro, es:

$$
\left[1+i \cdot \frac{K_{0}}{K} \cdot \frac{t c^{*}}{B \cdot K} \cdot \frac{\partial j}{\partial p_{q}} \cdot \frac{p_{k}^{*}}{B}\right] \cdot \frac{\partial j}{\partial t c^{*}}=\frac{\partial j}{\partial p_{q}} \cdot \frac{p_{k}^{*}}{B} \cdot\left[1+i \cdot \frac{K_{0}}{K}\right]+\frac{\partial j}{\partial W} \cdot \frac{\partial W}{\partial t c^{*}}+\frac{\partial j}{\partial \Pi} \cdot \frac{\partial \Pi}{\partial t c^{*}}
$$

Sustituyendo (3.III.) en esta ecuación, resulta:

$$
\left[1+i \cdot \frac{K_{0}}{K} \cdot \frac{t c^{*}}{B \cdot K} \cdot \frac{\partial j}{\partial p_{q}} \cdot \frac{p_{k}^{*}}{B}\right] \cdot \frac{\partial j}{\partial t c^{*}}=\frac{\partial j}{\partial p_{q}} \cdot \frac{p_{k}^{*}}{B} \cdot\left[1+i \frac{K_{0}}{K}\right]+\frac{\partial j}{\partial W} \cdot \frac{w}{B \cdot c} \cdot \frac{\partial j}{\partial t c^{*}}+\frac{\partial j}{\partial \Pi} \cdot \frac{\partial \Pi}{\partial t c^{*}}
$$

Sustituyendo ahora (3.II.) en esta expresión, tenemos:

$$
\begin{aligned}
& {\left[1+i \cdot \frac{K_{0}}{K} \cdot \frac{t c^{*}}{B \cdot K} \cdot \frac{\partial j}{\partial p_{q}} \cdot \frac{p_{k}^{*}}{B}\right] \cdot \frac{\partial j}{\partial t c^{*}}=\ldots} \\
& \ldots=\frac{\partial j}{\partial p_{q}} \cdot \frac{p_{k}^{*}}{B}\left[1+i \cdot \frac{K_{0}}{K}\right]+\frac{\partial j}{\partial W} \cdot \frac{w}{B \cdot c} \cdot \frac{\partial j}{\partial t c^{*}}+\frac{\partial j}{\partial \Pi} \cdot\left[i \cdot p_{k}^{*} \cdot\left(2 \cdot K-K_{0}\right)+\frac{i}{B} \cdot t c^{*} \cdot p_{k}^{*} \cdot 2 \cdot \frac{\partial j}{\partial t c^{*}}\right] \\
& {\left[1+i \cdot \frac{K_{0}}{K} \cdot \frac{t c^{*}}{B \cdot K} \cdot \frac{\partial j}{\partial p_{q}} \cdot \frac{p_{k}^{*}}{B}-\frac{\partial j}{\partial W} \cdot \frac{w}{B \cdot c}\right] \frac{\partial j}{\partial t c^{*}}=\ldots} \\
& \ldots=\frac{\partial j}{\partial p_{q}} \cdot \frac{p_{k}^{*}}{B} \cdot\left[1+i \cdot \frac{K_{0}}{K}\right]+\frac{\partial j}{\partial \Pi}\left[i \cdot p_{k}^{*} \cdot\left(2 \cdot K-K_{0}\right)+\frac{i}{B} \cdot t c^{*} \cdot p_{k}^{*} \cdot 2 \cdot \frac{\partial j}{\partial t c^{*}}\right] \\
& {\left[1+i \cdot \frac{K_{0}}{K} \cdot \frac{t c^{*}}{B \cdot K} \cdot \frac{\partial j}{\partial p_{q}} \cdot \frac{p_{k}^{*}}{B}-\frac{\partial j}{\partial W} \cdot \frac{w}{B \cdot c}\right] \frac{\partial j}{\partial t c^{*}}=\ldots} \\
& \ldots=\frac{\partial j}{\partial p_{q}} \cdot \frac{p_{k}^{*}}{B} \cdot\left[1+i \cdot \frac{K_{0}}{K}\right]+\frac{\partial j}{\partial \Pi} \cdot i \cdot p_{k}^{*} \cdot\left(2 \cdot K-K_{0}\right)+\frac{\partial j}{\partial \Pi} \cdot \frac{i}{B} \cdot t c^{*} \cdot p_{k}^{*} \cdot 2 \cdot \frac{\partial j}{\partial t c^{*}} \\
& {\left[1+i \cdot \frac{K_{0}}{K} \cdot \frac{t c^{*}}{B \cdot K} \cdot \frac{\partial j}{\partial p_{q}} \cdot \frac{p_{k}^{*}}{B}-\frac{\partial j}{\partial W} \cdot \frac{w}{B \cdot c}-\frac{\partial j}{\partial \Pi} \cdot i \cdot t c^{*} \cdot p_{k}^{*} \cdot \frac{2}{B}\right] \cdot \frac{\partial j}{\partial t c^{*}}=\ldots} \\
& \ldots=\frac{\partial j}{\partial p_{q}} \cdot \frac{p_{k}^{*}}{B} \cdot\left[1+i \cdot \frac{K_{0}}{K}\right]+\frac{\partial j}{\partial \Pi} \cdot i \cdot p_{k}^{*} \cdot\left(2 \cdot K-K_{0}\right)
\end{aligned}
$$

Por lo tanto, resulta:

$$
\frac{\partial j}{\partial t c^{*}}=\frac{\frac{\partial J}{\partial p_{q}} \cdot \frac{p_{k}^{*}}{B} \cdot\left(1+i \cdot \frac{K_{0}}{K}\right)+\frac{\partial j}{\partial \Pi} \cdot i \cdot p_{k}^{*} \cdot\left(2 \cdot K-K_{0}\right)}{1+i \cdot \frac{K_{0}}{K} \cdot \frac{t c^{*}}{B \cdot K} \cdot \frac{\partial j}{\partial p_{q}} \cdot \frac{p_{k}^{*}}{B}-\frac{\partial j}{\partial W} \cdot \frac{w}{B \cdot c}-\frac{\partial j}{\partial \Pi} \cdot i \cdot t c^{*} \cdot p_{k}^{*} \cdot \frac{2}{B}}
$$




\section{Deducción 7 \\ Deducción de los efectos de la variación del tipo de cambio real sobre el precio del bien $\underline{\text { manufacturado }}$}

Si la derivada de la demanda del bien manufacturado respecto del tipo de cambio real es negativa, la derivada del precio de dicho bien respecto del tipo de cambio real debe ser positiva. Para que el efecto de una subida del tipo de cambio real sobre el precio del bien manufacturado fuera negativo sería necesario que la elasticidad de la demanda respecto al tipo de cambio real no sólo fuera positiva sino que, además, fuera bastante mayor que uno:

$$
\begin{gathered}
\frac{\partial p_{q}}{\partial t c^{*}}=\frac{p_{k}^{*}}{B} \cdot\left[1+i \cdot \frac{K_{0}}{K} \cdot\left(1-\varepsilon_{Q^{d}, t c^{*}}\right)\right]<0 \Rightarrow\left[1+i \cdot \frac{K_{0}}{K} \cdot\left(1-\varepsilon_{Q^{d}, t c^{*}}\right)\right]<0 \\
\Rightarrow 1+i \cdot \frac{K_{0}}{K}-i \cdot \frac{K_{0}}{K} \cdot \varepsilon_{Q^{d}, t c^{*}}<0 \\
\Rightarrow 1+i \cdot \frac{K_{0}}{K}<i \cdot \frac{K_{0}}{K} \cdot \varepsilon_{Q^{d}, t c^{*}} \\
\Rightarrow \varepsilon_{Q^{d}, t c^{*}}>1+\frac{K}{i \cdot K_{0}}
\end{gathered}
$$

Deducción 8

Deducción de la derivada del PNN físico respecto del tipo de cambio real

Partiendo de la ecuación (25), que está expresada en términos monetarios, podemos obtener la expresión del $P N N$ con las variables físicas en unidades homogéneas de energía:

$$
\begin{gathered}
P N N=p_{y} \cdot Y^{d}+p_{q} \cdot Q^{d}+t c^{*} \cdot p_{y}^{*} \cdot X-\delta \cdot t c^{*} \cdot p_{k}^{*} \cdot K \\
P N N(F)=Y^{d}+Q^{d}+X-\delta \cdot K
\end{gathered}
$$

Sustituyendo en esta expresión la ecuación (8) $X=Y^{0}-Y^{d}-Y_{k}^{0}$, con explotación de toda la tierra disponible resulta:

$$
P N N(F)=Y^{0}-Y_{k}^{0}+Q^{d}-\delta . K
$$

Sustituyendo ahora las ecuaciones (14) $Q^{d}=j\left(p_{q}, W, \Pi\right)$ y (18) $K=\frac{1}{B} \cdot j\left(p_{q}, W, \Pi\right)$ y factoreando, tenemos:

$$
\operatorname{PNN}(F)=Y^{0}-Y_{k}^{0}+\left(1-\frac{\delta}{B}\right) j\left(p_{q}, W, \Pi\right)
$$

Derivando el Producto Nacional Neto físico respecto al tipo de cambio real resulta:

$$
\frac{\partial P N N(F)}{\partial t c^{*}}=\left(1-\frac{\delta}{B}\right) \cdot \frac{\partial j}{\partial t c^{*}}
$$

Deducción 9

Deducción de la derivada del $P N N$ nominal respecto del tipo de cambio real

Veamos ahora la situación con el efecto sobre la renta nacional contabilizada monetariamente, según la ecuación (25):

$$
P N N=p_{y} \cdot Y^{d}+p_{q} \cdot Q^{d}+t c^{*} \cdot p_{y}^{*} \cdot X-\delta \cdot t c^{*} \cdot p_{k}^{*} \cdot K
$$

Introduciendo la simplificación $p_{y}=t C^{*} \cdot p^{*}$ y sustituyendo según la ecuación (8) con explotación de toda la tierra disponible, $X=Y^{0}-Y^{d}-Y_{k}^{0}$, según la ecuación (14) $Q^{d}=j\left(p_{q}, W, \Pi\right)$ y según la ecuación (18) $K=\frac{1}{B} \cdot j\left(p_{q}, W, \Pi\right)$ y luego cancelando términos, tendremos:

$$
P N N=t c^{*} \cdot p_{y}^{*} \cdot\left(Y^{0}-Y_{k}^{0}\right)+p_{q} \cdot j\left(p_{q}, W, \Pi\right)-\delta \cdot t c^{*} \cdot p_{k}^{*} \cdot \frac{1}{B} \cdot j\left(p_{q}, W, \Pi\right)
$$

Derivando el $P N N$ en unidades monetarias respecto del tipo de cambio real, resulta:

$$
\frac{\partial P N N}{\partial t c^{*}}=p_{y}^{*} \cdot\left(Y^{0}-Y_{k}^{0}\right)+\frac{\partial p_{q}}{\partial t c^{*}} \cdot Q^{d}+p_{q} \cdot \frac{\partial j}{\partial t c^{*}}-\delta \cdot p_{k}^{*} \cdot \frac{Q^{d}}{B}-\delta \cdot t c^{*} \cdot \frac{p_{k}^{*}}{B} \cdot \frac{\partial j}{\partial t c^{*}}
$$




$$
\Rightarrow \frac{\partial P N N}{\partial t c^{*}}=p_{y}^{*} \cdot\left(Y^{0}-Y_{k}^{0}\right)+\left[\frac{\partial p_{q}}{\partial t c^{*}}-\delta \cdot \frac{p_{k}^{*}}{B}\right] \cdot Q^{d}+\left(p_{q}-\delta \cdot t c^{*} \cdot \frac{p_{k}^{*}}{B}\right) \cdot \frac{\partial j}{\partial t c^{*}}
$$

Deducción 10

Deducción la expresión general de la dinámica del crecimiento

Partiendo de la ecuación (25):

$$
P N N=p_{y} \cdot Y^{d}+p_{q} \cdot Q^{d}+t c^{*} \cdot p_{y}^{*} \cdot X-\delta \cdot t c^{*} \cdot p_{k}^{*} \cdot K
$$

Recurriendo a la simplificación $p_{y}=t c^{*} \cdot p_{y}^{*}$ y sustituyendo según la ecuación (8) con la especificación de la ecuación (1 bis), $X=Y^{0}-Y^{d}-Y_{k}^{0}$, sustituyendo además, por otro lado, según las ecuaciones (17) $Q^{d}=Q^{S}$ y (3 bis) $Q=B . K$ por otro lado, y factoreando tenemos:

$$
P N N=t c^{*} \cdot p_{y}^{*} \cdot\left(Y^{0}-Y_{k}^{0}\right)+\left(p_{q} \cdot B-\delta \cdot t c^{*} \cdot p_{k}^{*}\right) \cdot K
$$

A partir de esta expresión estudiaremos la dinámica del crecimiento derivando la misma con respecto al tiempo:

$$
\begin{aligned}
& \frac{d(P N N)}{d t}=\frac{d t c^{*}}{d t} \cdot p_{y}^{*} \cdot\left(Y^{0}-Y_{k}^{0}\right)+\left(\frac{d p_{q}}{d t} \cdot B-\delta \cdot \frac{d t c^{*}}{d t} \cdot p_{k}^{*}\right) \cdot K+\left(p_{q} \cdot B-\delta \cdot t c^{*} \cdot p_{k}^{*}\right) \cdot \frac{d K}{d t} \\
& \Rightarrow \frac{d(P N N)}{d t}=\frac{d p_{q}}{d t} \cdot B \cdot K+\left[p_{y}^{*} \cdot\left(Y^{0}-Y_{k}^{0}\right)-\delta \cdot p_{k}^{*} \cdot K\right] \cdot \frac{d t c^{*}}{d t}+\left(p_{q} \cdot B-\delta \cdot t c^{*} \cdot p_{k}^{*}\right) \cdot \frac{d K}{d t}
\end{aligned}
$$

Suponiendo que el sistema funciona con pleno empleo del capital instalado, $K_{0}=K$, la ecuación (10) es $p_{q}=\frac{\left[w+t c^{*} \cdot p_{k}^{*} \cdot c+i \cdot t c^{*} \cdot p_{k}^{*} \cdot c\right]}{B \cdot c}$. Derivando esta expresión del precio del bien manufacturado con respecto al tiempo resulta:

$$
\begin{aligned}
& \frac{d p_{q}}{d t}=\frac{d w}{d t} \cdot \frac{1}{B \cdot c}+\frac{d t c^{*}}{d t} \cdot \frac{p_{k}^{*}}{B}+\frac{d i}{d t} \cdot \frac{t c^{*} \cdot p_{k}^{*}}{B}+\frac{i \cdot p_{k}^{*}}{B} \cdot \frac{d t c^{*}}{d t} \\
& \Rightarrow \frac{d p_{q}}{d t}=\frac{d w}{d t} \cdot \frac{1}{B \cdot c}+\frac{d i}{d t} \cdot \frac{t c^{*} \cdot p_{k}^{*}}{B}+\left(\frac{p_{k}^{*}}{B}+\frac{i \cdot p_{k}^{*}}{B}\right) \cdot \frac{d t c^{*}}{d t} \\
& \Rightarrow \frac{d p_{q}}{d t}=\frac{d w}{d t} \cdot \frac{1}{B \cdot c}+\frac{d i}{d t} \cdot \frac{t c^{*} \cdot p_{k}^{*}}{B}+(1+i) \cdot \frac{p_{k}^{*}}{B} \cdot \frac{d t c^{*}}{d t}
\end{aligned}
$$

Reemplazando esta expresión en la derivada del $P N N$ respecto al tiempo y simplificando se obtiene:

$$
\begin{aligned}
& \frac{d(P N N)}{d t}=\left(\frac{d w}{d t} \cdot \frac{1}{c}+\frac{d i}{d t} \cdot t c^{*} \cdot p_{k}^{*}+(1+i) \cdot p_{k}^{*} \cdot \frac{d t c^{*}}{d t}\right) \cdot K+\ldots \\
& \ldots+\left[p_{y}^{*} \cdot\left(Y^{0}-Y_{k}^{0}\right)-\delta \cdot p_{k}^{*} \cdot K\right] \cdot \frac{d t c^{*}}{d t}+\left(p_{q} \cdot B-\delta \cdot t c^{*} \cdot p_{k}^{*}\right) \cdot \frac{d K}{d t}
\end{aligned}
$$

Reemplazando ahora la ecuación de $p_{q}$ y simplificando resulta:

$$
\begin{gathered}
\frac{d(P N N)}{d t}=\left(\frac{d w}{d t} \cdot \frac{1}{c}+\frac{d i}{d t} \cdot t c^{*} \cdot p_{k}^{*}+(1+i) \cdot p_{k}^{*} \cdot \frac{d t c^{*}}{d t}\right) \cdot K+\ldots \\
\ldots+\left[p_{y}^{*} \cdot\left(Y^{0}-Y_{k}^{0}\right)-\delta \cdot p_{k}^{*} \cdot K\right] \cdot \frac{d t c^{*}}{d t}+\left(\frac{w}{c}+t c^{*} \cdot p_{k}^{*}+i \cdot t c^{*} \cdot p_{k}^{*}-\delta \cdot t c^{*} \cdot p_{k}^{*}\right) \cdot \frac{d K}{d t}
\end{gathered}
$$

Factoreando y reordenando términos resulta:

$$
\begin{gathered}
\frac{d(P N N)}{d t}=\frac{d w}{d t} \cdot \frac{K}{c}+\frac{d i}{d t} \cdot t c^{*} \cdot p_{k}^{*} \cdot K+(1+i) \cdot p_{k}^{*} \cdot K \cdot \frac{d t c^{*}}{d t}+\ldots \\
\ldots+\left[p_{y}^{*} \cdot\left(Y^{0}-Y_{k}^{0}\right)-\delta \cdot p_{k}^{*} \cdot K\right] \cdot \frac{d t c^{*}}{d t}+\left[\frac{w}{c}+(1+i-\delta) \cdot t c^{*} \cdot p_{k}^{*}\right] \cdot \frac{d K}{d t}
\end{gathered}
$$




$$
\begin{gathered}
\frac{d(P N N)}{d t}=\frac{d w}{d t} \cdot \frac{K}{c}+\frac{w}{c} \cdot \frac{d K}{d t}+p_{k}^{*} \cdot t c^{*}\left(K \cdot \frac{d i}{d t}+(1+i-\delta) \frac{d K}{d t}\right) \\
+\left[p_{y \cdot}^{*} \cdot\left(Y^{0}-Y_{k}^{0}\right)+(1+i-\delta) p_{k}^{*} \cdot K\right] \cdot \frac{d t c^{*}}{d t}
\end{gathered}
$$

Deducción 11

Deducción de la inversión en función del crecimiento de la fuerza laboral

El pleno empleo laboral significa igualdad de la oferta y la demanda de trabajo:

$$
\text { w. }\left(L^{S}-L^{d}\right)=0
$$

Sustituyendo a partir de la ecuación (19) $L^{S}=L_{0} \cdot e^{\text {n.t }}$ y de la ecuación (20) $L^{d}=L_{q}+L_{y}^{0}, L_{y}^{0}=b . T^{0}, L_{q}=\frac{Q^{S}}{B . c}$, se obtiene:

$$
w \cdot\left(L_{0} \cdot e^{n . t}-\frac{Q^{S}}{B . c}-L_{y}^{0}\right)=0 \quad \Rightarrow . e^{n . t}=\frac{Q^{S}}{B . c}+L_{y}^{0}
$$

Sustituyendo ahora de acuerdo a las ecuaciones (17) $Q^{d}=Q^{S}$ y (3 bis) $Q=B K$, resulta:

$$
\begin{gathered}
L_{0} \cdot e^{n \cdot t}=\frac{K}{c}+L_{y}^{0} \\
K=c \cdot\left(L_{0} \cdot e^{n \cdot t}-L_{y}^{0}\right) \\
\Rightarrow \frac{d K}{d t}=c \cdot n \cdot L_{0} \cdot e^{n \cdot t}
\end{gathered}
$$

Deducción 12

Deducción del tipo de cambio real necesario para los objetivos del ajuste con el enfoque monetario de la balanza de pagos

El gobierno actuará operando sobre diversas variables (salarios, tasa de interés, tipo de cambio) de modo que la ecuación reducida de la dinámica del crecimiento se convierta en:

$$
\frac{d P N N}{d t}=P N N \cdot \pi=p_{k}^{*} \cdot t c^{*} \cdot K \cdot\left(1+i^{*}\right) \cdot \frac{d \pi^{e}}{d t}+\left[p_{y}^{*} \cdot\left(Y^{0}-Y_{k}^{0}\right)+(1+i-\delta) \cdot p_{k}^{*} \cdot K\right] \cdot \frac{d t c^{*}}{d t}
$$

A partir de la ecuación (7) $t c^{*}=t c \cdot \frac{p^{*}}{p}$, aplicando logaritmos y derivando respecto al tiempo, se obtiene:

$$
\frac{\frac{d t c^{*}}{d t}}{t c^{*}}=\frac{\frac{d t c}{d t}}{t c}-\frac{\frac{d p}{d t}}{p} \quad \Rightarrow \frac{d t c^{*}}{d t}=t c^{*}\left[\frac{\frac{d t c}{d t}}{t c}-\pi\right]
$$

Reemplazando esta expresión en la ecuación anterior y factoreando resulta:

$$
P N N \cdot \pi=\left\{p_{k}^{*} \cdot K \cdot\left(1+i^{*}\right) \cdot \frac{d \pi^{e}}{d t}+\left[p_{y}^{*} \cdot\left(Y^{0}-Y_{k}^{0}\right)+(1+i-\delta) \cdot p_{k}^{*} \cdot K\right] \cdot\left(\frac{\frac{d t c}{d t}}{t c}-\pi\right)\right\} \cdot t c^{*}
$$

Por otra parte, teniendo en cuenta que:

$$
P N N=t c^{*} \cdot p_{y}^{*} \cdot\left(Y^{0}-Y_{k}^{0}\right)+\left(p_{q} \cdot B-\delta \cdot t c^{*} \cdot p_{k}^{*}\right) \cdot K
$$

$\mathrm{Y}$ que en condiciones de pleno empleo del capital instalado es:

Reemplazando $p_{q}$ en $P N N$ y simplificando resulta:

$$
p_{q}=\frac{w}{B \cdot c}+\frac{t c^{*} \cdot p_{k}^{*}}{B}+\frac{i \cdot t c^{*} \cdot p_{k}^{*}}{B}
$$

$$
\begin{aligned}
P N N & =t c^{*} \cdot p_{y}^{*} \cdot\left(Y^{0}-Y_{k}^{0}\right)+\left(\frac{w}{c}+t c^{*} \cdot p_{k}^{*}+i \cdot t c^{*} \cdot p_{k}^{*}-\delta \cdot t c^{*} \cdot p_{k}^{*}\right) \cdot K \\
& \Rightarrow P N N=t c^{*} \cdot p_{y}^{*} \cdot\left(Y^{0}-Y_{k}^{0}\right)+\left[\frac{w}{c}+(1+i-\delta) \cdot t c^{*} \cdot p_{k}^{*}\right] \cdot K \\
& \Rightarrow P N N=t c^{*} \cdot\left[p_{y}^{*} \cdot\left(Y^{0}-Y_{k}^{0}\right)+(1+i-\delta) \cdot p_{k}^{*} \cdot K\right]+w \cdot \frac{K}{c}
\end{aligned}
$$


Reemplazando esta expresión en $P N N$. $\pi$, se obtiene:

$$
\begin{gathered}
\left\{t c^{*} \cdot\left[p_{y}^{*} \cdot\left(Y^{0}-Y_{k}^{0}\right)+(1+i-\delta) \cdot p_{k}^{*} \cdot K\right]+w \cdot \frac{K}{c}\right\} \cdot \pi=\ldots \\
\ldots=\left\{p_{k}^{*} \cdot K \cdot\left(1+i^{*}\right) \cdot \frac{d \pi^{e}}{d t}+\left[p_{y}^{*} \cdot\left(Y^{0}-Y_{k}^{0}\right)+(1+i-\delta) \cdot p_{k}^{*} \cdot K\right] \cdot\left(\frac{\frac{d t c}{d t}}{t c}-\pi\right)\right\} \cdot t c^{*}
\end{gathered}
$$

Distribuyendo, reordenando y factoreando surge:

$$
\begin{aligned}
& t c^{*} \cdot \pi \cdot\left\{p_{y}^{*} \cdot\left(Y^{0}-Y_{k}^{0}\right)+(1+i-\delta) \cdot p_{k}^{*} \cdot K\right\}-p_{k}^{*} \cdot K \cdot\left(1+i^{*}\right) \cdot \frac{d \pi^{e}}{d t} \cdot t c^{*}=\ldots \\
& \ldots= t c^{*} \cdot\left\{p_{y \cdot}^{*} \cdot\left(Y^{0}-Y_{k}^{0}\right) \cdot\left(\frac{\frac{d t c}{d t}}{t c}-\pi\right)+(1+i-\delta) \cdot p_{k}^{*} \cdot K \cdot\left(\frac{\frac{d t c}{d t}}{t c}-\pi\right)\right\}-\frac{w K}{c} \cdot \pi \\
& t c^{*} \cdot\left[\pi \cdot\left\{p_{y \cdot}^{*}\left(Y^{0}-Y_{k}^{0}\right)+(1+i-\delta) \cdot p_{k}^{*} \cdot K\right\}-p_{k}^{*} \cdot K \cdot\left(1+i^{*}\right) \cdot \frac{d \pi^{e}}{d t}\right]=\ldots \\
& \ldots= t c^{*} \cdot\left\{p_{y \cdot}^{*} \cdot\left(Y^{0}-Y_{k}^{0}\right) \cdot\left(\frac{\frac{d t c}{d t}}{t c}-\pi\right)+(1+i-\delta) \cdot p_{k}^{*} \cdot K \cdot\left(\frac{\frac{d t c}{d t}}{t c}-\pi\right)\right\}-\frac{w K}{c} \cdot \pi \\
& t c^{*} \cdot\left[\pi \cdot p_{y \cdot}^{*} \cdot\left(Y^{0}-Y_{k}^{0}\right)+\pi \cdot(1+i-\delta) \cdot p_{k}^{*} \cdot K-p_{k}^{*} \cdot K \cdot\left(1+i^{*}\right) \cdot \frac{d \pi^{e}}{d t}\right]=\ldots \\
& \ldots= t c^{*}\left\{p_{y}^{*}\left(Y^{0}-Y_{k}^{0}\right) \frac{\frac{d t c}{d t}}{t c}-p_{y}^{*}\left(Y^{0}-Y_{k}^{0}\right) \pi\right\} \\
& \ldots=t c^{*} \cdot\left\{p_{y \cdot}^{*} \cdot\left(Y^{0}-Y_{k}^{0}\right) \cdot \frac{\frac{d t c}{d t}}{t c}+(1+i-\delta) \cdot p_{k}^{*} \cdot K \cdot \frac{\frac{d t c}{d t}}{t c}\right\}-\frac{w \cdot K}{c} \cdot \pi \\
& t c^{*} \cdot\left[2 \cdot \pi \cdot p_{y \cdot}^{*}\left(Y^{0}-Y_{k}^{0}\right)+2 \cdot \pi \cdot(1+i-\delta) \cdot p_{k}^{*} \cdot K-p_{k}^{*} \cdot K \cdot\left(1+i^{*}\right) \cdot \frac{d \pi^{e}}{d t}\right]=\ldots \\
&\left.(1+i-\delta) p_{k}^{*} \cdot K \frac{\frac{d t c}{d t}}{t c}-(1+i-\delta) p_{k}^{*} \cdot K \cdot \pi\right\}-\frac{w \cdot K}{c} \pi
\end{aligned}
$$

$t c^{*} \cdot\left[\left\{p_{y}^{*} \cdot\left(Y^{0}-Y_{k}^{0}\right)+(1+i-\delta) \cdot p_{k}^{*} \cdot K\right\} \cdot\left(2 \cdot \pi-\frac{\frac{d t c}{d t}}{t c}\right)-p_{k}^{*} \cdot K \cdot\left(1+i^{*}\right) \cdot \frac{d \pi^{e}}{d t}\right]=-\frac{w \cdot K}{c} \cdot \pi$

Despejando $t c^{*}$ y reemplazando según $K=c . L_{0 . e^{n . t}}$, resulta el tipo de cambio real que satisface las condiciones de la política sugerida por F.M.I., o sea, el valor del tipo de cambio real que habría que alcanzar para lograr los objetivos del ajuste conforme al enfoque de la balanza de pagos:

$$
t c^{*}=\frac{\left[-w \cdot L_{0} \cdot e^{n \cdot t} \cdot \pi\right]}{\left[p_{y}^{*} \cdot\left(Y^{0}-Y_{k}^{0}\right)+(1+i-\delta) \cdot p_{k}^{*} \cdot c \cdot L_{0} \cdot e^{n \cdot t}\right] \cdot\left[2 \pi-\frac{\frac{d t c}{d t}}{t c}\right]-p_{k}^{*} \cdot\left(1+i^{*}\right) \cdot \frac{d \pi^{e}}{d t} \cdot c \cdot L_{0} \cdot e^{n . t}}
$$




\section{Demostración 1}

Demostración de la consistencia del tipo de cambio real si la tasa de inflación no puede sufrir aceleraciones y la tasa de devaluación/depreciación es completamente controlable por la autoridad monetaria.

Partiendo del valor necesario del tipo de cambio real para alcanzar el éxito en la política inspirada en el enfoque monetario de la balanza de pagos en situación de estanflación, tenemos:

$$
t c^{*}=\frac{-w \cdot L_{0} \cdot e^{n . t} \cdot \pi}{\left[p_{y \cdot}^{*}\left(Y^{0}-Y_{k}^{0}\right)+(1+i-\delta) \cdot p_{k^{*}}^{*} c . L_{0} \cdot e^{n . t}\right] \cdot\left[2 \cdot \pi-\frac{\frac{d t c}{d t}}{t c}\right]-p_{k^{*}}^{*}\left(1+i^{*}\right) \cdot \frac{d \pi^{e}}{d t} \cdot c \cdot L_{0} \cdot e^{n . t}}
$$

Si ocurre que:

$$
\frac{d \pi}{d t}=0 \quad, \quad \frac{d^{2} \pi^{e}}{d t^{2}}=0 \quad \frac{d\left(\frac{\frac{d t c}{d t}}{t c}\right)}{d t}=0
$$

entonces se podrá hallar el valor límite de $t c^{*}$ cuando el tiempo tiende a infinito siguiendo la regla de L’Hôspital. Aplicando dicha regla y factoreando resulta:

Simplificando, es:

$$
\lim _{t \rightarrow \infty} t c^{*}=\frac{-w \cdot L_{0} \cdot n \cdot e^{n \cdot t} \cdot \pi}{\left[(1+i-\delta) \cdot p_{k}^{*} \cdot\left(2 \cdot \pi-\frac{\frac{d t c}{d t}}{t c}\right)-p_{k}^{*} \cdot\left(1+i^{*}\right) \cdot \frac{d \pi^{e}}{d t}\right] \cdot c \cdot L_{0} \cdot n \cdot e^{n . t}}
$$

$$
\lim _{t \rightarrow \infty} t c^{*}=\frac{-w \cdot \pi}{\left[(1+i-\delta) \cdot p_{k}^{*} \cdot\left(2 \cdot \pi-\frac{\frac{d t c}{d t}}{t c}\right)-p_{k^{*}}^{*}\left(1+i^{*}\right) \cdot \frac{d \pi^{e}}{d t}\right] \cdot c}
$$

Dado que el numerador es negativo, el denominador también debe serlo, de lo contrario el valor límite del tipo de cambio real sería negativo, lo que es absurdo. Para obtener un valor consistente debe cumplirse la restricción:

$$
(1+i-\delta) \cdot p_{k}^{*} \cdot\left(2 \cdot \pi-\frac{\frac{d t c}{d t}}{t c}\right)<p_{k}^{*} \cdot\left(1+i^{*}\right) \cdot \frac{d \pi^{e}}{d t} \Rightarrow \frac{\frac{d t c}{d t}}{t c}>2 \cdot \pi-\frac{\left(1+i^{*}\right)}{(1+i-\delta)} \cdot \frac{d \pi^{e}}{d t}
$$

Si fuera 2. $\pi=\frac{\frac{d t c}{d t}}{t c}$, entonces el valor límite bien determinado del tipo de cambio real sería:

$$
\lim _{t \rightarrow \infty} t c^{*}=\frac{w \cdot \pi}{p_{k}^{*} \cdot\left(1+i^{*}\right) \cdot \frac{d \pi^{e}}{d t} \cdot c}
$$

\section{Deducción 13}

Se parte de la expresión hallada para el tipo de cambio real necesario para el éxito del ajuste basado en el enfoque monetario de la balanza de pagos en situación de estanflación:

$$
t c^{*}=\frac{-w \cdot L_{0} \cdot e^{n . t} \cdot \pi}{\left[p_{y}^{*} \cdot\left(Y^{0}-Y_{k}^{0}\right)+(1+i-\delta) \cdot p_{k^{*}}^{*} c \cdot L_{0} \cdot e^{n . t}\right] \cdot\left[2 \cdot \pi-\frac{\frac{d t c}{d t}}{t c}\right]-p_{k^{*}}^{*}\left(1+i^{*}\right) \cdot \frac{d \pi^{e}}{d t} \cdot c \cdot L_{0} \cdot e^{n . t}}
$$

Suponiendo que se cumple:

Los objetivos de la política de ajuste exigen:

$$
\frac{d \pi}{d t}=e^{P . t} \quad, \frac{\frac{d t c}{d t}}{t c}=e^{T C . t} \quad, \frac{d i}{d t}=\left(1+i^{*}\right) \cdot \frac{d \pi^{e}}{d t}=\left(1+i^{*}\right) \cdot e^{E . t}
$$

$$
T C>P \quad, \quad E>P
$$


Sustituyendo estas expresiones, en las que se permite la aceleración de la tasa de inflación, de la tasa de devaluación y de la tasa de crecimiento del tipo de interés nominal, en la ecuación del tipo de cambio real necesario para el ajuste basado en el enfoque monetario de la balanza de pagos resulta:

$$
t c^{*}=\frac{-w \cdot L_{0} \cdot e^{n . t} \cdot e^{P . t}}{\left[p_{y}^{*} \cdot\left(Y^{0}-Y_{k}^{0}\right)+(1+i-\delta) \cdot p_{k}^{*} \cdot c \cdot L_{0} \cdot e^{n . t}\right] \cdot\left[2 \cdot e^{P . t}-e^{T C . t}\right]-p_{k}^{*} \cdot\left(1+i^{*}\right) \cdot e^{E . t} \cdot c \cdot L_{0} \cdot e^{n . t}}
$$

Dividiendo el numerador y el denominador por $\mathrm{L}_{0} \cdot \mathrm{e}^{\mathrm{n} . \mathrm{t}}$ se convierte la expresión en:

$$
t c^{*}=\frac{-w \cdot e^{P . t}}{\frac{p_{y}^{*} \cdot\left(Y^{0}-Y_{k}^{0}\right) \cdot\left[2 \cdot e^{P . t}-e^{T C . t}\right]}{L_{0} \cdot e^{n . t}}+(1+i-\delta) \cdot p_{k}^{*} \cdot c \cdot\left[2 \cdot e^{P . t}-e^{T C . t}\right]-p_{k}^{*} \cdot\left(1+i^{*}\right) \cdot e^{E . t} \cdot c}
$$

Si dividimos ahora el numerador y el denominador por $e_{-w}^{P . t}$, resulta:

$$
t c^{*}=\frac{-w}{\frac{p_{y \cdot}^{*}\left(Y^{0}-Y_{k}^{0}\right) \cdot\left[2-\frac{e^{T C . t}}{e^{P . t}}\right]}{L_{0} \cdot e^{n . t}}+(1+i-\delta) \cdot p_{k}^{*} \cdot c \cdot\left[2-\frac{e^{T C . t}}{e^{P . t}}\right]-p_{k^{*}}^{*}\left(1+i^{*}\right) \cdot \frac{e^{E . t}}{e^{P . t}} \cdot c}
$$

Pasando los divisores a exponentes negativos y factoreando es:

$$
t c^{*}=\frac{-w}{p_{y}^{*} \cdot\left(Y^{0}-Y_{k}^{0}\right) \cdot L_{0}^{-1} \cdot\left(2 \cdot e^{-n \cdot t}-e^{(T C-P-n) \cdot t}\right)+(1+i-\delta) \cdot p_{k}^{*} \cdot c \cdot\left(2-e^{(T C-P) \cdot t}\right)-p_{k}^{*} \cdot\left(1+i^{*}\right) \cdot e^{(E-P) \cdot t} \cdot c}
$$

Partiendo de la expresión:

\section{Demostración 2}

$$
t c^{*}=\frac{-w}{p_{y}^{*} \cdot\left(Y^{0}-Y_{k}^{0}\right) \cdot L_{0}^{-1} \cdot\left(2 \cdot e^{-n \cdot t}-e^{(T C-P-n) \cdot t}\right)+(1+i-\delta) \cdot p_{k}^{*} \cdot c \cdot\left(2-e^{(T C-P) \cdot t}\right)-p_{k}^{*} \cdot\left(1+i^{*}\right) \cdot e^{(E-P) \cdot t} \cdot c}
$$

La obtención de un valor determinado en el límite, cuando $t$ tiende a infinito, depende de la relación entre las tasas de los factores exponenciales.

Si suponemos, en una primera aproximación, que las tasas a las que crecen exponencialmente el tipo de cambio nominal, la tasa de inflación y el tipo de interés nominal son iguales entre sí, $P=T C=E$, tendríamos:

$$
\begin{gathered}
t c^{*}=\frac{-w}{p_{y}^{*} \cdot\left(Y^{0}-Y_{k}^{0}\right) \cdot L_{0}^{-1} \cdot e^{-n \cdot t}+(1+i-\delta) \cdot p_{k}^{*} \cdot c-p_{k^{*}}^{*}\left(1+i^{*}\right) \cdot c} \\
\lim _{t \rightarrow \infty} t c^{*}=\frac{-w}{\left(i-i^{*}-\delta\right) \cdot p_{k}^{*} \cdot c}
\end{gathered}
$$

Para que esta expresión tenga sentido económico es necesario que el denominador sea negativo, o sea, que

$$
i-i^{*}-\delta<0
$$

Sin embargo, no sólo no hay ninguna seguridad de que esto pueda cumplirse, sino que, especialmente cuando existen niveles de alta inflación que crecen exponencialmente, lo normal es esperar que esta expresión sea mayor que cero. En ese caso, el tipo de interés nominal es mayor que uno (tiende a ser mayor o igual que la inflación) y muy superior en valor absoluto al tipo de interés real, cuyo valor normal es aproximadamente del orden de $i^{*} \cong 0,02$, llegando en condiciones excepcionales a ser una tasa anual negativa. Estas realidades harían que el denominador fuera positivo y, en consecuencia, que el valor límite del tipo de cambio real resultara un absurdo desde el punto de vista económico.

Además, existe un valor de $i$ que convierte en cero al denominador dejando totalmente indeterminado el valor del tipo de cambio real, inclusive en el límite cuando $t$ tiende a infinito. 
Por otra parte, aun cuando el denominador fuera negativo y existiera un valor definido del tipo de cambio real con sentido económico, cabe recordar que eso procedería de haber supuesto que la tasa de incremento de la devaluación era igual a la tasa de crecimiento de la inflación, con lo cual el tipo de cambio real no sufre ninguna mejora. Lo mismo ocurre con la tasa de crecimiento del tipo de interés nominal, que hemos supuesto igual a la de la tasa de inflación, lo que implica que no existe mejora del tipo de interés real.

La persistencia en los objetivos de la política económica del ajuste propiciado por el enfoque monetario de la balanza de pagos exige que:

Suponiendo por ahora que:

$$
T C>P, E>P
$$

$$
T C-P-n<0
$$

Entonces tenemos que a partir de la expresión:

$$
t c^{*}=\frac{-w}{p_{y}^{*} \cdot\left(Y^{0}-Y_{k}^{0}\right) \cdot L_{0}^{-1} \cdot\left(2 \cdot e^{-n \cdot t}-e^{(T C-P-n) \cdot t}\right)+(1+i-\delta) \cdot p_{k}^{*} \cdot c \cdot\left(2-e^{(T C-P) \cdot t}\right)-p_{k}^{*} \cdot\left(1+i^{*}\right) \cdot e^{(E-P) \cdot t} C}
$$

En el límite, resulta una expresión del tipo:

$$
\lim _{t \rightarrow \infty} t c^{*}=\frac{w}{\frac{C_{1}}{\infty}-\frac{C_{2}}{\infty}+C_{3}-C_{4} . \infty-C_{5} . \infty}=0
$$

Donde las $C_{i}$ son valores numéricos constantes.

Esta conclusión no se modifica si ocurriera que: $T C-P-n \geq 0$ 